\title{
Les mouvements de subsistance et le problème de l'économie morale sous l'ancien régime et la Révolution française
}

\section{Cynthia Bouton}

\section{(2) OpenEdition \\ 1 Journals \\ Édition électronique \\ URL : https://journals.openedition.org/ahrf/104 \\ DOI : $10.4000 /$ ahrf.104 \\ ISSN : 1952-403X \\ Éditeur : \\ Armand Colin, Société des études robespierristes}

\section{Édition imprimée}

Date de publication : 1 mars 2000

Pagination : 71-100

ISSN : 0003-4436

\section{Référence électronique}

Cynthia Bouton, « Les mouvements de subsistance et le problème de l'économie morale sous l'ancien régime et la Révolution française ", Annales historiques de la Révolution française [En ligne], 319 | janvier-mars 2000, mis en ligne le 11 mai 2006, consulté le 22 avril 2022. URL : http:// journals.openedition.org/ahrf/104; DOI : https://doi.org/10.4000/ahrf.104

Ce document a été généré automatiquement le 22 avril 2022

Tous droits réservés 


\title{
Les mouvements de subsistance et le problème de l'économie morale sous l'ancien régime et la Révolution française ${ }^{1}$
}

\author{
Cynthia Bouton
}

1 Depuis le XVIIe siècle et jusqu'au milieu du xIxe, les mouvements de subsistance prirent un caractère significatif et presque constant de la vie politique des pays européens. Parmi eux, la France se distingue par sa longue tradition de troubles de subsistance. Elle en éprouva aussi le plus grand nombre. Les crises les plus importantes sont maintenant bien connues $^{2}$ et le nombre d'émeutes est impressionnant. Guy Lemarchand et Jean Nicolas ont identifié plus de 1400 émeutes de subsistance pour la période 1661-1789 et John Markoff en a dénombré plus de 1200 entre 1788 et 1793. La crise de l'an III en produisit plus de 200. Les mouvements persistèrent jusqu'au milieu du xixe siècle, avec plus de 1300 émeutes $^{3}$. On constate qu'il y eut au moins une émeute par an entre la crise de 1709 et le Consulat. Et, comme nous le savons tous, la guerre du blét $^{4}$ se développa au point de devenir une question politique capable de contribuer à la chute de ministres, à la désacralisation de la monarchie, et au sort des gouvernements révolutionnaires.

2 Dans cet article, j'analyserai deux dimensions de l'histoire de l'approvisionnement en France. Je partirai tout d'abord des conditions qui ont favorisé les émeutes de subsistance. Pour préciser le contexte de ces mouvements, je remonterai à une époque antérieure à la Révolution. Ensuite, je passerai en revue l'histoire des émeutes de subsistance sous l'Ancien Régime et pendant la période révolutionnaire, en précisant les objectifs et le comportement des émeutiers, la réponse des autorités, des magistrats, et de la force armée. Quoique plusieurs historiens, comme Georges Lefebvre et George Rudé, aient remarqué la maturité croissante des comportements des émeutiers, tous, à l'exception notable de Guy Lemarchand, ont eu tendance à insister sur leurs traits communs ${ }^{5}$. Certes, il y avait des ressemblances, surtout dans les motivations, mais les 
différences sont importantes : en effet, les émeutiers répondaient par des tactiques adaptées aux conditions spécifiques créées par les revirements dans la politique économique du gouvernement ou par les changements de longue durée dans le commerce des grains et dans les structures sociales, par exemple. Je présenterai enfin un tableau des émeutiers en tant qu'agents jouant des rôles plus actifs que passifs dans leurs vies ${ }^{6}$.

Le droit à la subsistance: l'histoire et l'économie morale

3 Les anthropologues ont souvent remarqué le caractère central d'un droit à la subsistance dans les sociétés paysannes. Une perception de la nourriture comme bien social se trouve dans la plupart des sociétés paysannes, sinon toutes. Cette perception entraînait la proposition suivante : étant donné que la faim est une réalité quotidienne mais que la nourriture, comme la terre qui la produit, est bornée en quantité, chacun a droit à une part des ressources limitées de la nature. La reconnaissance générale de droits sociaux sur les objets de première nécessité rendait illégitime tout effort de nier le droit à la subsistance à qui que ce soit et exigeait qu'on secourût ceux qui en avaient besoin ${ }^{7}$. Le refus de suivre ces normes pouvait provoquer et légitimer l'indignation, voire des protestations violentes.

4 En effet, cette norme était généralement admise au Moyen Âge, même si les propriétaires disputèrent parfois le moment choisi et l'étendue des revendications sur leurs biens ${ }^{8}$. Les achats et ventes sur les marchés restaient limités, informels, visibles et protégés par des relations sociales de voisinage. Les marchés fonctionnaient comme des espaces à la fois économiques et sociaux ${ }^{9}$. Les mauvaises récoltes pouvaient réduire les réserves disponibles, mais la capacité d'une personne ou d'une communauté à bénéficier d'une portion de ce qu'il restait était déterminée, en partie, par les relations sociales et les normes ${ }^{10}$.

5 Vers la fin du xvie siècle, il apparut dans le royaume de France une expression historique du droit à la subsistance, ce que E. P. Thompson appela, pour l'Angleterre, l'économie morale ${ }^{11}$. Pour Thompson, l'économie morale était une notion de légitimité qui désignait des fonctions économiques propres, liées au droit le plus général à la subsistance. La proposition que les grains et leurs produits étaient un bien d'intérêt commun, un bien social, restait largement reconnue, même par les détenteurs de biens et de pouvoir ${ }^{12}$. L'effectivité de cette reconnaissance se révèle dans le complexe des régulations locales et royales qui enveloppait la production et la distribution des grains et de leurs dérivés ${ }^{13}$. Cette économie morale s'exprimait surtout dans un contexte local et communal structuré par des relations empreintes de paternalisme et d'inégalité. Les rapports entre les élites et le menu peuple, entre les seigneurs et leurs tenanciers, entre les autorités municipales et les habitants, entre les souverains et leurs sujets, engageaient des revendications mutuelles des deux parties: par exemple, ce que les élites voyaient comme un acte de charité, le peuple le percevait comme un droit $^{14}$. Les deux parties essayaient d'utiliser le système de paternalisme à leur propre avantage. Ainsi, le modèle paternaliste était devenu le cadre historique dans lequel s'exprimait l'économie morale de l'Ancien Régime, et l'économie morale, à son tour, le cadre dans lequel s'exprimait le droit à la subsistance ${ }^{15}$.

6 Alors que l'économie morale structurait la manière dont on comprenait les problèmes quotidiens de subsistance, elle ne pouvait cependant, par sa seule force, provoquer les émeutes de subsistance. Certes, il y eut quelques émeutes au Moyen Âge, mais elles restèrent rare $^{16}$. Ce furent les comportements spécifiques résultant du développement 
du commerce des grains qui, en entrant en conflit avec l'économie morale, suscitèrent les émeutes. Les changements économiques, sociaux et politiques des xvie et xvire siècles créèrent, en effet, un contexte favorable aux émeutes frumentaires, entraînant à la fois une augmentation dans le nombre des désordres et un élargissement des régions susceptibles de s'émouvoir. Des crises importantes se déclenchèrent en 1585-86, 1630-31, et 1660-62. Toutes furent accompagnées d'émeutes de subsistance. Cette période est mieux connue pour les troubles religieux et les désordres anti-fiscaux, bien sûr, pourtant, à la fin du Xvire siècle, surtout après la crise de 1692-94, les émeutes de subsistance devinrent alors la forme la plus fréquente de désordre.

Les émeutes de subsistance sous l'Ancien Régime

7 Que s'était-il passé ? La première moitié du xvie siècle fut une période d'expansion générale ${ }^{17}$, et c'était l'expansion elle-même qui créait des pressions nouvelles sur la production et le système de distribution en France. Les villes s'accroissaient et pressuraient les arrières-pays producteurs: le nombre de consommateurs non producteurs se multipliait et les besoins de l'armée, en augmentant, pesaient sur les réseaux de ravitaillement ${ }^{18}$. Ainsi transportait-on davantage de grains entre les producteurs et les consommateurs des villes ou de l'armée.

8 Les années 1560 marquèrent un tournant dans les fortunes en France ${ }^{19}$. La population croissante dépassait les ressources, les structures de production et les moyens traditionnels de distribution. Une période d'inflation se déclencha et on vit les prix augmenter plus rapidement que les salaires. La polarisation de la société s'accéléra et la France connut une paupérisation grandissante. Le " petit âge glaciaire » débuta dans les années 1580 et se poursuivit jusqu'au milieu du xixe siècle, réduisant la productivité agricole. Les progrès de la religion réformée et la faiblesse de la monarchie depuis la mort de Henri II, ouvrirent presque un siècle de guerres civile et internationale. La situation en France se dégrada davantage dans les années 1630. La guerre de Trente Ans exigea d'énormes approvisionnements et, à l'exception d'un bref répit entre 1659 et 1667, le Royaume resta en guerre pendant le reste du siècle. La cadence des révoltes paysannes et urbaines contre les exigences fiscales de l'État s'accéléra. Malgré l'augmentation de la productivité agricole pendant la première moitié du xviıe siècle, dans quelques régions comme le Bassin parisien ${ }^{20}$, les prix montèrent parfois à des niveaux effrayants à la suite de mauvaises récoltes ${ }^{21}$. Après 1650 , la productivité agricole diminua ${ }^{22}$.

9 Ces changements bouleversèrent les structures des marchés traditionnels. L'arrivée d'acheteurs nouveaux entraîna souvent une forte hausse des prix et l'épuisement des marchés. Les comportements des marchands contrariaient la tradition, comportements qui deviendront fréquents pendant la Révolution : par exemple, des achats de quantités énormes, à n'importe quel prix, soit sur les marchés, soit chez les producteurs, et surtout des comportements désignés sous les noms de spéculation et d'accaparement. Les régions pauvres en espèces se trouvaient incapables de faire concurrence à celles qui en disposaient. Les mauvaises récoltes aggravaient la situation. De plus, quand une disette de grains coïncidait avec une disette d'espèces ou de crédit pour les achats, une communauté et même toute une région en pâtissaient.

10 En effet, plusieurs sortes de marchés coexistaient. À côté des marchés locaux visibles du Moyen Âge, apparurent les marchés invisibles du grand commerce. Ce nouveau commerce trouvait son origine dans une combinaison de plusieurs formes de développement. Par exemple, la monarchie elle-même cherchait à libérer la circulation 
des grains. Tout d'abord, la guerre presque continuelle exigeait la recherche constante de subsistances pour les troupes. De plus, l'accroissement de Paris conduisait à rechercher des subsistances de plus en plus éloignées de la capitale. Pendant la crise des années 1660, par exemple, des commissionnaires parisiens cherchèrent des quantités énormes de grains jusque dans le Languedoc, les accumulèrent à Bordeaux et les envoyèrent au Havre pour atteindre Paris ${ }^{23}$. Pour faciliter ces grands achats et ces transports à grande distance, la monarchie devait briser la résistance des municipalités et des parlements qui tentaient de protéger les besoins locaux et régionaux, ainsi que leurs privilèges bien sûr. L'intendant de Guyenne réussit à surmonter la résistance du parlement de Bordeaux et fit sortir de son port des grains pour Paris ${ }^{24}$. Selon Guy Lemarchand, la monarchie réduisit le rôle du parlement de Rouen dans l'approvisionnement de son ressort, pendant la crise des années $1690^{25}$. À partir du xvire siècle, l'État luttait constamment contre les autorités locales qui essayaient de protéger les habitants, en limitant un commerce de plus en plus élargi des grains. En réalité, la monarchie privilégiait des droits de propriété au détriment du droit local à la subsistance.

11 Les commissionnaires qui approvisionnaient l'armée, Paris et les autres villes, parvinrent à élargir le rayon d'action de leur commerce. Ils battaient la campagne, payaient des prix énormes et drainaient des grains loin de leurs réseaux traditionnels. Certes, ni la monarchie ni les magistrats des grandes villes ne prétendaient diriger euxmêmes un système d'approvisionnement devenu complexe. Ainsi, au lieu de le faire eux-mêmes ils le confiaient à des marchands. Pendant les graves crises des années 1660 et 1690 , les marchands rencontrèrent moins de restrictions dans leurs activités que durant les années précédentes ${ }^{26}$. Malgré le désir de la monarchie de restreindre les abus trop évidents des marchands ${ }^{27}$, les besoins des troupes et de Paris limitaient son intervention. En effet, l'État facilitait la spéculation en même temps qu'il cherchait à développer la circulation des grains et, ce qui est même plus important, il la facilitait davantage pendant les périodes de crise ${ }^{28}$.

Entre la fin du xvie siècle et les années 1690 , les émeutes de subsistance devinrent de plus en plus fréquentes. Le peuple résistait aux ravitailleurs des troupes et de Paris et des autres négociants quand ils menaçaient leur droit à la subsistance. Les émeutes de cette période prenaient une forme plutôt ad hoc et désordonnée. Le type d'émeute le plus fréquent était l'arrêt des convois de grains pendant lequel les participants se distribuaient les grains, tandis que les émeutes dans les marchés restaient rares. Par contre, le peuple faisait de fréquentes perquisitions dans les magasins où les marchands stockaient leurs achats, ou contraignait les autorités à le faire et à séquestrer les vivres trouvés pour les vendre au prochain marché.

Les émeutiers de cette première période recouraient assez souvent aux actes de violence. Des bagarres éclataient entre le peuple et les marchands, parfois les autorités : on donnait des coups de poing, on déchirait les vêtements, on menaçait de tuer avec des couteaux et des bâtons. Les émeutiers attaquaient aussi la propriété : les maisons des marchands de grains étaient mises à sac, les meubles et les marchandises détruits. William Beik a proposé récemment d'intégrer ces formes d'expression de colère collective dans le cadre d'une "culture de rétribution ", signifiant qu'il "n'est pas suffisant de corriger un prix, de redresser un abus, ou d'expulser un coupable, et que l'on doit chercher les responsables lorsque les normes ne sont plus respectées ${ }^{29}$ 

nombre sont bien connus des historiens de la Révolution. Elles suspendaient l'exportation, faisaient des perquisitions, offraient des primes et effectuaient des achats pour leurs habitants. Ces tactiques permettaient d'améliorer le ravitaillement local, mais risquaient aussi d'aggraver la situation ailleurs. Les magistrats invitaient à d'autres efforts : la charité, les greniers communaux, le rationnement, les ateliers pour chômeurs, parfois la taxation du prix des grains ou de celui du pain ${ }^{30}$. Le gouvernement menaçait d'une répression sévère, mais en fin de compte, le nombre des arrestations était faible et les condamnations rares. Quelques châtiments exemplaires, typiques de l'Ancien Régime, étaient infligés, mais le plus souvent on ne poursuivait pas les émeutiers. L'emploi de la force resta exceptionnel pour plusieurs raisons : tout d'abord, l'armée se trouvait souvent en opération, mais aussi l'arrivée des troupes limitait le pouvoir local des magistrats, enfin la présence des soldats était loin d'être un facteur d'ordre public ${ }^{31}$ ! diverses de la population: des paysans, des ouvriers, des artisans, des bourgeois aussi. Bien sûr, les paysans, les ouvriers et les artisans dominaient lors des émeutes, mais les habitants plus aisés y participaient et donnaient souvent leur voix en leur faveur. Malgré leur répugnance pour le désordre, les autorités locales faisaient parfois cause commune avec les habitants, n'hésitant pas à se saisir de l'éclat de l'émeute pour justifier leur intervention en faveur de la communautée ${ }^{32}$. En général, et parce qu'il existait toujours un accord large sur le droit à la subsistance, les mouvements frumentaires attiraient des alliés divers. taxations populaires plus disciplinées et relativement non violentes du xvwe siècle. En effet, les actes de taxation populaire se produisaient très rarement. Le peuple voyait les comportements nouveaux dans le commerce des grains comme des actes d'injustice individuelle, comme une déviance de quelques individus et répondaient à cette conduite avec l'intention de punir les coupables. C'est seulement plus tard que l'on commença à comprendre que ce qui se passait faisait partie d'un développement plus répandu et que l'on élabora des tactiques nouvelles. Ainsi, le peuple savait adapter ses réponses aux conditions historiques particulières, même si leurs origines appartenaient au droit à la subsistance ou à l'économie morale. Quand les émeutiers répétaient "qu'ils préféraient être pendus, ou tués, que de mourir de faim ", ils exprimaient leur volonté de faire tout ce qui était nécessaire pour protéger leurs droits.

Presque un siècle plus tard, dans les années 1760 et 1770, le caractère des mouvements de subsistance avait beaucoup changé. Après 1750, un accroissement lent de la productivité agricole est sensible dans le Bassin parisien ${ }^{33}$. Le Royaume était devenu plus commerçant et plus industriel, mais seulement dans certaines régions, comme dans les centres maritimes ou manufacturiers. On ne s'étonnera pas que ces centres, dont Paris, aient été les plus riches et qu'ils soient devenus d'importants marchés de grains et de farines ${ }^{34}$. En 1760, deux économies au moins coexistaient en France : une économie locale et régionale qui souffrait de plus en plus de l'accroissement d'une économie nationale naissante ${ }^{35}$.

Cette période éprouva aussi une révolution démographique. Grâce aux accroissements, même modestes, de productivité et aux améliorations dans les réseaux de communication et de transport, peu de gens mouraient directement de la faim.

Annales historiques de la Révolution française, 319 | janvier-mars 2000 
Pourtant, un plus grand nombre de personnes vivaient dans la pauvreté, sinon dans la misère ${ }^{36}$. La période connut des mouvements défavorables des prix et des salaires ${ }^{37}$ et une crise croissante dans les structures de l'assistance des pauvres ${ }^{38}$.

Ce fut aussi une époque de réformes, surtout dans le commerce des grains. La vision des Physiocrates l'avait emporté sur celle des « libéraux égalitaires » dont a parlé Simone Meyssonnier ${ }^{39}$. Le système des Physiocrates avait séduit l'imagination des détenteurs du pouvoir. Les réformes sont bien connues: la monarchie déclara la liberté économique en 1763 , la supprima en 1770 , s'y engagea à nouveau en 1774 , et recula encore en 1776. Ainsi, quatre fois en treize ans, le roi changea-t-il de politique.

La politique de commerce libre des grains ne signifiait pas une rupture aussi nette avec le passé que le croyaient ses partisans ${ }^{40}$. Bien sûr, la monarchie avait depuis longtemps facilité les efforts des marchands pour mieux approvisionner ses armées et Paris. Les marchands et les producteurs qui se comportaient depuis longtemps d'une façon ambiguë se trouvaient de plus en plus en opposition avec l'esprit de l'économie morale. Pourtant, cette politique représenta un changement important dans la façon dont la monarchie se concevait elle-même d'une part, et concevait ses relations avec ses sujets d'autre part ${ }^{41}$. L'Arrêt du 13 septembre 1774, rédigé par Turgot, expliquait que l'on pourrait satisfaire les besoins du pays de deux manières: «ou par l'agence du commerce lui-même, ou par l'intervention du gouvernement». La monarchie se déclarait désormais incapable de le faire aussi bien que les marchands.

21 Auparavant, le gouvernement prétendait favoriser le consommateur au détriment du propriétaire de grains et surtout du marchand, mais avec cette déclaration, il changeait de côté. Tandis qu'il jurait d'aider ses sujets nécessiteux, il s'engageait à protéger le droit de propriété des producteurs et des marchands. Les interdictions d'intervention dans le commerce des grains signifiaient qu'il protégerait en premier lieu ceux qui vendaient les subsistances. En effet, la monarchie invitait tous ses sujets à se faire marchands de grains et promettait «de les soutenir avec une protection visible». L'Arrêt de 1774 dénonça les stratégies antérieures comme « illusoires ». Que ce système entraînerait des bienfaits pour les consommateurs restait, à vrai dire, une question de foi.

Le choix du gouvernement en faveur de la liberté du commerce des grains contribua à transformer, à son tour, la façon dont le peuple concevait la monarchie. Un gouvernement qui, pendant des siècles, avait affirmé sa préoccupation de soulager et d'approvisionner son peuple, rompait les manifestations les plus visibles de son engagement. Le peuple pouvait voir et interpréter lui-même les résultats. Le RoiBoulanger avait trahi son peuple. Comme l'a résumé Steven Kaplan, « directement ou indirectement, la liberté du commerce nourrissait et empirait la crise parce qu'elle créa une atmosphère fébrile de spéculation, ouvrit des débouchés nouveaux pour le commerce, désordonna le système de distribution et répandit la peur et l'incertitude $\aleph^{42}$. Les années 1760 et 1770 connurent une série de récoltes médiocres, sans doute, mais elles avaient été aggravées par les perturbations causées par les producteurs et les marchands livrés à eux-mêmes pour découvrir les moyens de servir leurs propres intérêts.

Les formes et la cadence des mouvements populaires se modifièrent. Ces changements répondaient en partie au revirement dans la politique du gouvernement et aux développements du système d'approvisionnement. Par rapport à la période précédente, le nombre d'émeutes doubla presque entre 1763 et 1789 , et prit des formes nouvelles. 
Les émeutiers montraient une compréhension beaucoup plus fine du système d'approvisionnement et du caractère plus structurel que contingent auquel ils étaient confrontés. Les arrêts de convois de grains et de farines restaient le type le plus fréquent des désordres, sauf pendant la Guerre des farines. Mais les émeutes sur les marchés, les attaques de magasins, et les incursions chez les fermiers et laboureurs prirent une place de plus en plus importante. Par ailleurs, les émeutiers recouraient beaucoup plus souvent à la taxation populaire. Ces configurations nouvelles se manifestèrent le plus visiblement pendant les années 1770.

L'augmentation du nombre d'émeutes sur les marchés et de taxations populaires était liée à la liberté illimitée du commerce. La fonction des marchés, comme celle du gouvernement, changeaient dans le nouveau système de commerce le marché devenait le centre des préoccupations populaires. C'était parce que les Arrêts du gouvernement interdisaient aux autorités locales d'intervenir sur les prix des grains, que le peuple se décidait à le faire lui-même ${ }^{43}$. Il comprenait que c'était au marché que les prix devaient se former, que c'était là qu'on se rassemblerait pour le faire. C'était souvent au marché que le peuple apportait les grains saisis ailleurs. Ainsi, la place du marché devint-elle un enjeu central pour le mouvement populaire, et assez tard dans l'histoire des émeutes de subsistance, au moment même où ce type de marché perdait en réalité de son importance. Bien sûr, des actes de taxation eurent lieu ailleurs aussi : dans des fermes, des greniers, des boulangeries, lors des arrêts des convois, mais le peuple cherchait à restituer au marché un rôle central, voire un rôle qui semble ne pas même lui avoir appartenu au Moyen Âge.

25 Un autre caractère important se révèle dans l'explosion du nombre d'émeutes au domicile des laboureurs et des fermiers, surtout pendant la Guerre des farines. Ce type d'émeute fut une réponse adaptée à la fois à la politique nouvelle et à la situation économique et sociale en pays de grande culture et fut très fréquent pendant la période révolutionnaire, comme au xixe siècle. Ces désordres rassemblaient les manouvriers agricoles et les petits et moyens exploitants contre les gros détenteurs de grains. Les émeutiers prirent soin de choisir comme cibles non seulement ceux qu'ils soupçonnaient d'accaparer, de spéculer et de mépriser leur droit à la subsistance, mais aussi ceux qui créaient d'autres sources de tension dans leur milieu ${ }^{44}$. Ils choisissaient les plus gros propriétaires exploitants, les grands fermiers qui concentraient les marchés de terre, ou ceux qui étaient liés au régime seigneurial. Ainsi les émeutiers se déchaînèrent-ils contre l'élite agricole qui profitait du système de liberté illimitée du commerce et symbolisait le pouvoir, au cœur de leurs communautés.

Dans les années 1760-70 les mouvements de subsistance évoluèrent beaucoup. Un siècle auparavant ces actions désordonnées et violentes visaient plutôt la punition des responsables que la protection des droits, parce qu'alors, on personnalisait les comportements commerciaux. Maintenant les revendications portaient sur les produits et les processus d'approvisionnement. La violence vindicative était rare.

27 Par ailleurs, le caractère de la participation changeait aussi. Les émeutiers venaient exclusivement des couches populaires de la société. Les journaliers, les compagnons, les artisans marginaux dans les villes, les manouvriers et les vignerons dans la campagne formaient le pivot des mouvements. Surtout à la campagne, les manouvriers agricoles jouaient un rôle de premier plan lors des visites chez les fermiers. Par contre, les bourgeois et les propriétaires des villes avaient largement disparu des rangs des émeutiers et même de leurs partisans. Cette évolution était due à l'essor de la 
population ouvrière et artisanale, vulnérable aux changements du système d'approvisionnement de la seconde moitié du xviIIe siècle, ainsi qu'au refus croissant que les élites opposaient aux émeutes de subsistance comme moyen légitime ou même admissible de combattre ces changements: le nombre de ceux qui rejetaient la proposition de l'économie morale augmentait. Selon Colin Lucas «les propriétaires comprenaient moins et craignaient plus la foule $»^{45}$. Certes, les mouvements de subsistance visaient alors la propriété.

Cette période instaura une politique de répression qui influencera les réponses ultérieures: les troupes de ligne et les cours prévôtales furent invitées à soutenir la politique de liberté économique. Tandis que la maréchaussée s'occupait des arrestations, les troupes "pacifiaient" la population. L'armée fournissait aussi des soldats pour protéger les convois de grains. Par exemple, les troupes escortèrent les bateaux de grains tout au long de la Garonne et de la Dordogne durant les crises de 1770 et $1773^{46}$. L'intensité d'une émeute à Reims, en 1770 , effraya tellement que les autorités réquisitionnèrent des troupes qui restèrent en ville pendant plus $d^{\prime} u n a^{47}$.

Le recours à l'armée fut le plus évident pendant la Guerre des farines. Quand les désordres se multiplièrent dans le Bassin parisien, la monarchie prit des mesures sévères ${ }^{48}$. Au total, 25000 soldats vinrent maintenir l'ordre et prévenir de nouveaux désordres sur les marchés ${ }^{49}$. Bien que la Guerre des farines eût atteint son paroxysme le 18 mai, le calme ne revint pas complètement. Les soldats occupèrent les villes du Bassin parisien jusqu'en novembre ${ }^{50}$.

30 Le roi réserva le jugement des émeutiers aux cours prévôtales. La loi de 1670 avait déjà confié ce genre de procès aux cours prévôtales, mais la Guerre des farines fut la première occasion qui permit à la monarchie de faire fonctionner cette juridiction unique $^{51}$. La Guerre des farines entraîna l'arrestation de 548 personnes et de nombreuses condamnations, dont deux à mort. Tout ceci témoigne d'une intervention de l'État sur une échelle très importante. Comme Florence Gauthier l'a observé pour la Révolution, « la main invisible d'Adam Smith était devenue la main très visible de la loi martiale $»^{52}$

La monarchie et ses ministres attribuaient la persistance des émeutes de subsistance à l'ignorance populaire. Le peuple, prétendaient-ils, ne comprenait pas ses propres intérêts. Ainsi essayèrent-ils de le rééduquer. Chaque arrêt, chaque déclaration mettant en œuvre la politique de liberté du commerce, était accompagné d'un discours sur les bienfaits de la nouvelle politique. Des justifications semblables accompagnaient les instructions aux intendants, subdélégués, officiers municipaux et parlements ${ }^{53}$. La monarchie chargeait ses officiers d'instruire le peuple, de lui expliquer la liberté économique et de lui montrer ses propres intérêts ${ }^{54}$. Même si le roi hésitait dans son engagement à la politique de liberté économique, il persista dans sa mission de rééducation. La déclaration de 1787, qui réaffirma la liberté du commerce, expliqua ainsi ses hésitations précédentes : «il n'est pas rare que les vérités politiques aient besoin de temps et de discussion pour arriver à maturité ». La déclaration concluait : "c'est maintenant qu'il faut fixer les principes " ${ }^{55}$. Les discours et les baïonnettes étaient nécessaires pour remettre le peuple sur le bon chemin et lui montrer les vérités économiques et politiques. Pourtant, en l'espace de deux ans, le gouvernement hésiterait une nouvelle fois.

Les émeutes de subsistance pendant la Révolution 

politiques. On sait bien qu'une ambiance de crise, mauvaises récoltes, disette, hausse des prix et désordres, accompagnait les mouvements de réforme et les débats législatifs. Le peuple et ses représentants devaient s'adapter aux conditions nouvelles et, à cause de ces adaptations, ils innovèrent beaucoup. John Markoff a récemment comparé le mouvement populaire des années 1788-1793, et en particulier le mouvement antiféodal, avec la politique révolutionnaire de la même période. Il trace la chronologie des revendications populaires et de la législation nationale, et montre clairement comment le peuple et ses représentants agirent en interaction et adaptèrent leurs réponses ${ }^{56}$. Les formes des mouvements révolutionnaires de subsistance, tout en ressemblant à bien des égards aux mouvements précédents et particulièrement à ceux des années 1760-1770, révèlent aussi une remarquable capacité d'adaptation aux changements de la période, ainsi qu'un véritable effort pour influencer la politique nationale. J'esquisserai ici à grands traits les mouvements de 1788-1789, 1792-1793, et 1794-1795.

En 1788-1789, une politisation intense produite par les luttes entre les parlements et la monarchie, la convocation des États généraux, la rédaction des cahiers de doléances et les élections, la Grande Peur et les débats sur le féodalisme, créa un climat électrique. Les magistrats locaux virent leur autorité défiée lorsque les bases de leur pouvoir commencèrent à s'effondrer. La liberté économique fut mise en cause à nouveau et des centaines de troubles de subsistance éclatèrent.

On trouve de nombreuses ressemblances entre ces émeutes et celles de la période précédente : les arrêts de convois étaient les plus fréquents, mais des émeutes sur les marchés et des attaques de greniers gardaient leur importance. Les incursions chez les producteurs ne furent pas aussi fréquentes que durant la Guerre des farines, mais conservèrent leur importance, surtout dans les pays de grande culture. Les émeutes dans les boulangeries restaient rares, mais se produisirent dans les grandes villes. Les taxations, enfin, l'emportaient sur les saisies de grain.

enoins, dans leur plus grande sensibilité aux autres questions politiques et dans une certaine tendance à se mêler à d'autres formes de protestation, les troubles de 1788-1789 marquèrent un changement important avec le passé. On note, par exemple, que la politique municipale avait partie liée avec les problèmes d'approvisionnement en 1789. Le bourg de Vernon connut des tensions multiples durant l'été et l'automne. Tandis que le contrôle de la municipalité était contesté par deux factions différentes, le peuple s'en prit à un négociant connu pour sa participation au commerce des grains. Les subsistances commandaient l'attention des contestataires ${ }^{57}$. Ces émeutes rejoignaient parfois d'autres revendications sur les impôts, les municipalités et les droits seigneuriaux par exemple. Le 23 juillet 1789 à Chartres, des «bandits" réclamèrent «la diminution du prix du grain et après l'avoir obtenu, ils se sont jetés sur les barrières et ont détruit les loges des commis, pillé leurs maisons et celles du directeur des fermes $\aleph^{58}$. On vit aussi réapparaître une violence vindicative, bien qu'assez rarement. Les émeutiers de Bourges attaquèrent le 27 juillet 1789 la maison d'un homme accusé de spéculer sur la farine et menacèrent de mettre le feu à plusieurs autres ${ }^{59}$.

La participation ressemblait à celle des années précédentes : ouvriers urbains et ruraux, vignerons, compagnons et artisans. Malgré la sévérité de cette crise, la participation des bourgeois et des autres groupes propriétaires restait toujours très faible. Par

Annales historiques de la Révolution française, 319 | janvier-mars 2000 
contre, on aperçoit fréquemment une collaboration ouverte entre les autorités et les émeutiers, même avant la révolution municipale. Par exemple, les consuls de Bergerac arrêtèrent un chargement de grains destinés à Périgueux, en mars 1789, pour l'usage de leurs propres habitants. Ils affichèrent l'explication suivante: «la nécessité n'a pas besoin de loi. $»^{60}$

L'intensité de la répression restait très variable. Parfois la sévérité est remarquable. Une émeute à Cherbourg, en juillet 1789, se termina par l'arrestation de 219 personnes. La cour condamna deux hommes à mort, trois aux galères à vie, cinq à un exil définitif, un à la prison à perpétuité, et un aux galères pour dix ans. La même cour condamna cinq femmes à être fouettées, puis à la prison à perpétuité61. Ces peines, cependant, furent exceptionnelles et très peu d'émeutes furent suivies de condamnations. La période accentuait les ambiguïtés que l'on rencontrait déjà dans les mouvements de subsistance. Au printemps 1789, l'intendant de Tours se plaignit du laxisme des cours de justice. Il écrivit que «quelques exemples seraient nécessaires...mais la variété des principes des juges de tous ces petits lieux, leur mollesse, leur habitude de céder à la crainte et aux considérations particulières, leur ignorance...toutes ces circonstances rendent leurs démarches incertaines et contradictoires $»^{62}$. Il suggéra que le gouvernement confie les jugements aux cours prévôtales, comme par le passé. Et, le 21 mai, le roi le fit, mais rien ne prouve que l'efficacité ou la régularité de la répression en aient été changées.

Pourtant, en 1788-1789, la maréchaussée et les troupes de ligne avaient tiré sur plus d'hommes et de femmes que durant toutes les émeutes de subsistance précédentes. Un carnage considérable suivit deux émeutes en avril et en septembre 1789 à Orléans. À chaque fois, les forces de répression tuèrent plusieurs personnes et en blessèrent maintes autres ${ }^{63}$. La présence des troupes témoigne non seulement de l'intervention croissante du gouvernement dans les affaires locales, mais aussi de l'incapacité des communautés à résoudre leurs propres problèmes. Les tensions autour de l'approvisionnement divisaient les communautés dans lesquelles s'effaçait la reconnaissance générale du droit à la subsistance. De plus en plus souvent, les villes, bourgs, et villages qui se déchiraient devaient recourir à la médiation de l'État pour rétablir la paix par la force armée.

Le 29 août 1789, l'Assemblée nationale décréta la liberté du commerce. La loi martiale du 21 octobre fut sa réponse à la poursuite des émeutes de subsistance ${ }^{64}$. Pourtant, en dépit de ce pouvoir, les autorités locales hésitaient à faire usage de la loi contre les troubles frumentaires. Les municipalités restaient sur des bases précaires, les nouveaux officiers partageaient parfois avec le peuple la même suspicion à l'égard de la liberté du commerce $^{65}$, tandis que la Révolution posait des questions fondamentales sur la nature des droits. Cette ambiguïté devint de plus en plus nette dans la seconde période de crise, en 1792-1793.

Les conditions se dégradèrent rapidement après la médiocre récolte de 1791. Les prix montèrent et la chute de l'assignat, qui perdit $40 \%$ de sa valeur nominale durant l'été 1792, aggrava la crise. Les cultivateurs, protégés par la loi sur la liberté du commerce, refusaient les assignats et hésitaient à fournir les marchés, même quand les autorités les y invitaient. Après la déclaration de guerre en avril 1792, les fournisseurs aux armées disputaient aux consommateurs les grains peu abondants ${ }^{66}$. La guerre se faisant sur le territoire français, les départements frontaliers subirent les ravages de l'ennemi et ceux des troupes françaises, et toutes sortes de perturbations dans les transports. 
Paris, qui restait une capitale "gâtée", pesait lourdement sur les réseaux d'approvisionnement. La récolte de 1792 fut meilleure, mais le système d'approvisionnement était déjà perturbé.

41 La Législative puis la Convention girondine défendirent obstinément la liberté du commerce, mais les consommateurs, et de plus en plus fréquemment leurs représentants locaux, demandaient d'autres solutions. De l'automne 1791 au printemps 1793, la France ne fut plus qu'une immense émeute de subsistance ${ }^{67}$. C'est alors que ces mouvements se différencièrent nettement de ceux de l'Ancien Régime et des premières années de la Révolution.

Tout d'abord, la plupart des événements eurent lieu sur les marchés. Le peuple, qui demandait une baisse des prix, concentrait ses actions sur la place du marché et cherchait à lui restituer sa fonction de centre de distribution et de transaction des subsistances: l'idée était de lui rendre son ancienne fonction d'espace social. Deuxièmement, un nombre croissant d'émeutiers s'adressaient aux administrateurs. Les habitants présentaient leurs plaintes devant les officiers de la municipalité, du district ou, parfois, du département. Ils exigeaient que les autorités prennent en compte leurs demandes de taxer les subsistances, de faire approvisionner les marchés, ou de punir les comportements estimés illégitimes. Troisièmement, l'organisation de ces mouvements était souvent préparée par le peuple, parfois en collaboration avec ses représentants locaux. F. Évrard et $\mathrm{M}$. Vovelle ont décrit des événements spectaculaires. Au printemps 1792, des bandes de cinq à quinze mille personnes, souvent armées et précédées par les tambours, parcouraient les marchés de la Beauce et forçaient les autorités à taxer les grains et les autres denrées de première nécessité ${ }^{68}$. Les participants à ces mouvements étaient des pauvres et souvent des chômeurs ruraux : des manouvriers, des ouvriers de la manufacture rurale, et bien sûr, des vignerons et de petits exploitants. Dès leur arrivée au marché ils trouvaient des alliés parmi les citadins menacés par la cherté et la disette.

En effet, le mouvement populaire, en 1792, avait développé un programme économique centré sur la question du droit à la subsistance dans le cadre du droit à l'existence. Ce n'était plus l'économie morale dont E. P. Thompson a parlé parce que celle-ci se structurait dans un contexte d'inégalité politique, de dépendance et de paternalisme. Les participants aux mouvements de cette période étaient, en principe, des citoyens investis de droits égaux qui attendaient que leurs représentants les écoutent et leur répondent ${ }^{69}$. De plus, ils essayaient de légitimer leurs revendications en rédigeant des pétitions et des procès-verbaux, dont ils exigeaient la validation en les faisant signer par les autorités. Ces documents écrits donnaient aussi une permanence aux revendications, en exprimant le désir que les taxations dureraient au-delà des actions qui les avaient imposées et préparèrent le programme du Maximum ${ }^{70}$. Par exemple, le 15 juillet 1793, une manifestation violente éclata devant le conseil municipal d'Amiens et les habitants forçèrent les membres à signer une promesse de faire baisser les prix. L'émeute commença hors de la salle de réunion du directoire du département de la Somme à Amiens, une foule de 1200 femmes demanda du pain. Le directoire décida alors de prendre soixante sacs de farine des réserves militaires pour les vendre aux habitants. Quelques jours plus tard, un marché se trouvant insuffisamment fourni, des femmes se plaignirent qu'on exportait pour l'armée. Un autre groupe d'habitants manifesta devant le Conseil ${ }^{11}$. 
Le peuple comprenait de mieux en mieux son pouvoir de faire des lois correspondant à ses besoins. Un tambour de la garde nationale de Chamarande, d'où partaient des émeutiers pour Étampes, en mars 1792, expliqua : «si les décrets ne veulent pas qu'ils mangent du pain, ils ne veulent pas les lois $»^{72}$. Un émeutier de Dourdan exprima les mêmes sentiments : « la loi n'était pas bonne, elle était mal faite, elle ne leur convenait pas, et il leur en fallait d'autres $»^{73}$. Ainsi, les pétitions et les procès-verbaux offraient de quoi élaborer de nouvelles lois qui prendraient en compte le droit à la subsistance que le peuple, maintenant investi de la souveraineté, pouvait faire reconnaître ${ }^{74}$.

Un quatrième caractère distinguait les émeutes de cette période des précédentes. Il s'agit du souci de réglementer les producteurs, un souci déjà exprimé durant la Guerre des farines, et maintenant plus affirmé encore. Puisque les cultivateurs et les marchands désertaient les marchés et que la loi de la liberté du commerce avait lié les mains des autorités, le peuple décida de réapprovisionner les marchés eux-mêmes. Les émeutiers firent irruption chez les producteurs, perquisitionnèrent leurs greniers et, au lieu de distribuer le grain entre eux comme ils le faisaient auparavant, ils exigèrent que les producteurs s'engagent à porter leur grain au marché. Parfois, ils insistaient pour obtenir des engagements écrits des cultivateurs ${ }^{75}$.

La crise ouvrit des fissures entre les différents niveaux de l'administration. Bien que l'Assemblée nationale s'attachât obstinément à la liberté du commerce, les opinions au niveau local étaient moins claires. La participation des autorités locales aux mouvements indique non seulement que le peuple voulait valider ses revendications de façon autre, en forçant la participation de ses représentants, mais signifie aussi que maints officiers municipaux, et parfois ceux du district, partageaient la vision de leurs concitoyens. Effectivement, ils avaient plus en commun avec le peuple qu'avec la plupart des députés. De plus, la décentralisation au niveau des municipalités et des districts donnait beaucoup de pouvoir à ces administrateurs en matière de subsistance ${ }^{76}$. Quand, en août 1793, le district de Cany, en Seine-Inférieure, arrêta une cargaison de 1400 quintaux de grains destinés à Paris, les officiers s'expliquèrent ainsi : « la faim fait taire la loi. $»^{77}$

Les relations entre les différents niveaux d'autorités et le peuple révèlent les difficultés posées par des propositions divergentes et parfois contradictoires qui persistaient sur la question des subsistances. On vit aussi réapparaître le recours à la répression et à la rééducation qui avaient caractérisé les décennies physiocratiques. Mais cette fois, les autorités devaient tenir compte de la rhétorique révolutionnaire de liberté et d'égalité. Par exemple, le 12 octobre, après plusieurs désordres dans le bourg de Montrelais, le commissaire du département de la Loire-Inférieure fit rassembler les habitants et leur fit prêter serment de "maintenir la liberté, l'égalité et la libre circulation des grains ». Dans son rapport au département, il prétendit que « le peuple a applaudi au serment et l'a répété avec transport. $»^{78}$

La réticence fréquente des gardes nationales, et parfois même des troupes, à participer à la répression des mouvements de subsistance touche aussi le nœud du problème. Par exemple, ni la garde nationale ni les troupes n'obéirent aux réquisitions à Dunkerque, en février 1792, et même après la proclamation de la loi martiale, elles refusèrent de marcher contre les émeutiers ${ }^{79}$.

Au niveau de l'Assemblée nationale les contradictions pesaient fortement. Chaque année, des décrets menaçaient de peines de plus en plus sévères ceux qui s'opposaient à la libre circulation des grains. Pourtant, après la chute de la monarchie et au milieu des 
orages des massacres de septembre, l'Assemblée législative décréta le 3 septembre 1792 une amnistie pour tous les faits relatifs aux lois sur le commerce des grains depuis le 14 juillet $1789^{80}$. Mais les administrateurs pouvaient en venir rapidement à la répression. Le 8 décembre 1792, la Convention girondine réaffirma sa politique de liberté du commerce et menaça de mort ceux qui entraveraient l'approvisionnement, et en particulier celui de Paris ${ }^{81}$. Pourtant, elle se contredit encore une fois en février 1793, après l'exécution du roi, en décidant une nouvelle amnistie pour tous les délits relatifs aux subsistances jusqu'au 21 janvier précédent ${ }^{82}$.

En effet, les révolutionnaires se trouvaient pris dans un dilemme qu'ils avaient euxmêmes créé. Ce dilemme reposait sur la question des Droits de l'homme : le droit à la subsistance et le droit à l'insurrection d'une part et le droit à la propriété privée absolue d'autre part. Les révolutionnaires, unis contre la tyrannie, se déchiraient dans un conflit entre des théories économiques et politiques opposées. La Révolution avait légitimé le droit à l'insurrection, mais ensuite elle devait distinguer entre le mouvement légitime et son contraire. Les émeutes de subsistance posaient directement ces questions et révélaient les contradictions.

51 Durant l'an II on chercha un équilibre avec la mise en place du programme du Maximum. Jean-Pierre Gross a souligné récemment que la proposition jacobine du partage, "à chacun sa part ", cherchait non à abolir la propriété mais à faire accepter que le droit à l'existence vienne limiter l'exercice du droit de propriété ${ }^{83}$. Selon cette proposition un « droit égal à la liberté » devait permettre d'éliminer la dépendance qui, selon Voltaire lui-même, venait de la misère ${ }^{84}$. Les Jacobins affirmaient que «le droit à l'existence devait avoir la priorité sur le droit à la satisfaction individuelle $»^{85}$. JeanPierre Gross analyse ainsi l'expérience : « Il n'en reste pas moins que la décision prise de socialiser la boulangerie et de collectiviser, non les moyens de prodution, mais les moyens de distribution, entraîne la mise en place d'un véritable communisme de communication ${ }^{86}$. La volonté de rendre les échanges visibles et sociaux est manifeste dans la mise en place des greniers d'abondance, la fabrication d'un pain de l'égalité, la cuisson dans les fours communaux. Pour combattre "l'égoïsme " on fit appel à une armée révolutionnaire ${ }^{87}$ et on eut recours au rationnement ${ }^{88}$. Leur intention de " fraternité » prenait une signification concrète en complément de la liberté et de l'égalité. La loi martiale fut supprimée le 23 juin 1793. Et en effet, il y eut une diminution importante dans la fréquence des émeutes de subsistance, et surtout des troubles de taxation; les «entraves à la circulation» apparaissent encore, mais sporadiquement pendant cette période ${ }^{89}$.

53 En décembre 1794, la Convention thermidorienne abrogea formellement le Maximum, supprima la nécessité d'avoir des acquits-à-caution pour les transports de subsistances et mit fin aux procès contre les accapareurs ${ }^{90}$. Pourtant, elle conserva les réquisitions tandis qu'on attendait impatiemment le retour aux marchés des marchands, attirés par la possibilité de faire un profit ${ }^{91}$. En dépit de l'insistance des autorités pour que les opérations se fassent sur les marchés, les marchands les désertèrent, confirmant leur déclin. Pourtant, durant tout l'an III, la Convention elle-même poursuivit en vain ses efforts pour les réanimer ${ }^{92}$.

54 À la fin de l'année 1794, la situation économique s'était beaucoup dégradée. L'assignat entamait sa chute finale vers une valeur quasi nulle ${ }^{93}$. L'hiver fut rigoureux et les rivières gelèrent, stoppant les transports dans le Nord. La guerre empêchait toujours 
l'accès aux réseaux traditionnels d'importation. Les prix montèrent en flèche ${ }^{94}$. Malgré la poursuite des réquisitions et le maintien de la taxe sur le pain, le gouvernement se contentait de protéger les marchands au lieu de protéger les consommateurs. Richard Cobb a décrit cette "politique incohérente du gouvernement de l'an III ». En effet, il nota que cette "année de la faim» avait laissé «dans l'imagination populaire » un souvenir «bien plus profond que celui des rigueurs dirigées de l'époque de la Terreur $»^{95}$. La récolte de 1795 fut meilleure en certains endroits, mais dans d'autres la production avait diminué ${ }^{96}$.

derniers mois de 1794 et le printemps de 1795 connurent un accroissement du nombre d'émeutes de subsistance, surtout dans le Bassin parisien et en Normandie. On voit comment les circonstances de cette période, les changements dans la législation, les comportements économiques et les conditions sociales pouvaient influer sur le caractère du mouvement populaire.

Très peu de troubles éclatèrent sur les marchés, fort dégarnis à cette époque, ou dans les magasins des marchands. Par contre, les arrêts de convois et les incursions chez les cultivateurs devinrent les formes les plus caractéristiques. Malgré la vigilance des colonnes volantes, les émeutiers arrêtaient beaucoup de convois de grain. La plupart des émeutes dans les fermes ressemblaient à celles de la Guerre des farines. Pourtant, il y avait autour de Paris et dans le pays de Caux des rassemblements importants d'hommes, de femmes et d'enfants qui venaient de plusieurs communes et battaient la campagne pour effectuer des réquisitions ${ }^{97}$. Parfois, les mouvements servaient à la fois à intercepter les transports et à soumettre les cultivateurs à leurs réglementations : le peuple cherchait surtout à garder, sur place, les provisions locales.

Les manifestations devant les autorités étaient presque aussi fréquentes que les attaques contre les convois et les fermes. Ces comportements se comprennent si on tient compte des conditions auxquelles le peuple était confronté : les marchés, non fournis, ne pouvaient plus fonctionner; la résistance aux réquisitions conduisait les consommateurs désespérés à chercher leur subsistance chez les producteurs; les autorités, n'étant plus celles de l'an II, se refusaient à taxer les prix; le peuple se plaignait de leur insensibilité et manifestait devant eux.

Pour la première fois depuis la fin du xvire siècle, les émeutiers de l'anIII pillèrent davantage grains, farines et pain qu'ils ne les taxèrent. Par exemple, les émeutiers d'Amiens prirent, sans payer, la farine qu'ils trouvèrent dans un grenier du ci-devant évêché ${ }^{8}$. À Yvetôt, des « escadrons » de 80 à 100 hommes prirent le grain qu'ils avaient trouvé dans les fermes de cinq cultivateurs de la commune de Grenouille ${ }^{99}$. Précisons, toutefois, qu'il est très difficile de savoir avec certitude si les émeutiers ont saisi le grain ou s'ils l'ont payé : pendant cette période, les forces de l'ordre étaient tellement hostiles aux émeutiers qu'elles qualifiaient indistinctement de pillage ce qui pouvait être une taxation.

De plus, les mouvements révélaient un niveau de violence qui ressemblait à celui des xvie et xvire siècles ${ }^{100}$. Cependant, les émeutiers dirigeaient leur rage contre les autorités et rarement contre les marchands ou les cultivateurs. Le peuple se sentait trahi par ses représentants. On entendait encore une rhétorique de fraternité sans qu'une législation ne lui donnât consistance. Par exemple, un arrêté du 17 septembre 1795 du directoire du département de la Côte-d'Or rappela aux cultivateurs que "l'obligation d'approvisionner [en grains] ceux qui en ont besoin est commune à tous ceux qui en détiennent...ce devoir est inséparable du droit sacré de la propriété $»^{101}$. 
Pourtant les administrateurs ne faisaient pas grand-chose pour faire rentrer les cultivateurs dans leur devoir. Aussi, les émeutiers agressaient parfois les autorités, surtout les officiers municipaux, quand ils ne répondaient pas à leurs demandes ${ }^{102}$.

L'abrogation du Maximum donna naissance à une nouvelle interprétation des mouvements frumentaires. Le gouvernement thermidorien élimina progressivement l'ambiguïté qui l'avait entourée jusque-là : il décriminalisait de plus en plus les comportements des marchands et criminalisait ceux des consommateurs. Les lois de mars et juin 1795 décrétèrent à nouveau la répression des émeutes de subsistance ${ }^{103}$. La Constitution de l'an III supprima la clause qui reconnaissait « le droit à l'insurrection » et rendit la loi martiale permanente. Elle abolit les districts, «la charnière entre le département et la populace ${ }^{104}$, mais ce changement affaiblit considérablement la capacité de l'administration locale à jouer un rôle significatif dans l'approvisionnement.

61 La Convention thermidorienne effaçait aussi toute retenue concernant l'intervention de l'armée dans les désordres intérieurs. De plus en plus souvent, elle fit appel aux troupes pour disperser les émeutes et protéger les transports. Il fallut quatre bataillons pour apaiser des émeutes à Rouen au début d'avril $1795^{105}$. À partir d'avril 1795,les troupes patrouillaient dans le Bassin parisien, en juillet le gouvernement créa une armée de l'intérieur chargée de maintenir l'ordre, réprimer les troubles et protéger les convois ${ }^{106}$. Après mai 1795 , une garde nationale à caractère censitaire fut constituée pour disposer d'une force plus fiable. De nombreuses municipalités faisaient garder militairement les marchés ${ }^{107}$. L'an III témoigne de la création d'un système répressif important. Bien sûr, l'intensité de la répression dépendait de la volonté des autorités locales et de celle des troupes, et cette volonté variait beaucoup. Guy Lemarchand a remarqué que les administrateurs de la Seine-Inférieure hésitaient à recourir à la répression, mais les représentants en mission les y forçaient ${ }^{108}$. Jean-Paul Bertaud a cité l'exemple des carabiniers obligés de quitter Paris, en mai 1795, parce qu'ils «ne tueraient pas des hommes demi-morts de faim " s'ils se soulevaient pour avoir du pain $^{109}$. Néanmoins, l'attitude du gouvernement indiquait clairement que la chance avait tourné pour ceux qui réclamaient le droit à la subsistance.

Après 1795-1796, les émeutes de subsistance disparurent quasiment ou se mêlèrent à la mendicité et au brigandage. Les récoltes de 1796 et 1797 furent meilleures. Le gouvernement abandonna définitivement les billets de confiance en février 1797. L'approvisionnement des marchés se rétablit lentement, mais les réquisitions persistaient en plusieurs endroits. Le prix du grain diminua lentement ${ }^{110}$. Vers la fin de 1796, le gouvernement réanima un peu l'assistance publique locale ${ }^{111}$. La France avait alors moins d'ennemis et le ravitaillement de l'armée en fut allégé d'autant. La défaite de la Contre-Révolution libéra la capacité productive de l'Ouest. Malgré un niveau de brigandage très élevé, les réseaux de distribution se reconstituaient ${ }^{112}$. Pourtant, les autorités durent recourir parfois aux réquisitions, même pendant les dernières années du Directoire ${ }^{113}$. La récolte de 1798 rendit l'approvisionnement difficile pendant l'hiver et le printemps de 1799 .

En octobre 1795, le gouvernement réactiva une loi de l'Ancien Régime, qui rendait la commune responsable en cas d'atteinte à la propriété sur son territoire. Cette loi visa, en particulier, les entraves à la circulation des grains ${ }^{114}$. Enfin, en juin 1797, une nouvelle expérience de liberté du commerce des grains fut votée, autorisant les ventes 
et achats n'importe où, sous réserve que les marchands obtiendraient une patente ${ }^{115}$. Le Directoire maintint toutefois l'interdiction d'exporter.

À l'exception d'une courte période de crise en 1812, qui conduisit Napoléon à instaurer une forme de maximum, ces lois fondèrent la réponse des gouvernements du xixe siècle aux mouvements de subsistance. Mais les mouvements avec leurs revendications d'un droit à la subsistance se poursuivaient. Et comme les précédents, ceux qui suivirent adaptèrent leurs réponses aux conditions qui se présentaient.

\section{NOTES}

1.Cette étude fait partie d'un projet collectif intitulé « The Politics of Provisioning : England, France and Germany from the sixteenth to the nineteenth centuries ", mené avec John Bohstedt, Manfred Gailus, et Martin Geyer. Il s'agit d'une histoire comparée de la politique d'approvisionnement et de la réponse populaire en Angleterre, en France et en Allemagne, du xvie au xixe siècle. Nous avons reçu des subventions du Council of European Studies de Columbia University, du National Endowment for the Humanities, et de la Humbolt-Stiftung Foundation. J'ai aussi reçu des bourses de mon université, Texas A \& M University. Je voudrais les en remercier. J’ai présenté cette intervention à l'Assemblée de la Société des études robespierristes, le 8 juin 1997, grâce à l'aimable invitation de Monsieur le Professeur Jean-Paul Bertaud, que je remercie vivement. Je tiens à exprimer ma profonde gratitude à Florence Gauthier pour sa lecture attentive et ses observations.

2.Les émeutes les plus importantes éclatèrent en 1585-86, 1630-31, 1661-62, 1692-94, 1709-10, 1725, 1738-42, 1747-48, 1757, 1760, 1770-72, 1773, 1775, 1788-89, 1792, 1795, 1802, 1811-12, 1816-17, 1829-30, 1846-47, 1852-54. La bibliographie des travaux sur le sujet des émeutes de subsistance est trop abondante pour être présentée ici, mais les notes infrapaginales en signaleront quelques-uns. Ma contribution au projet collectif cité plus haut comprendra les références bibliographiques, accompagnées de cartes et de tableaux détaillant les résultats présentés ici.

3.Guy Lemarchand, «Troubles populaires au XviIIe siècle et conscience de classe : une préface à la Révolution française ", Annales historiques de la Révolution française, $\mathrm{n}^{\circ} 279$, 1990, pp. 32-48 ; une communication de Jean Nicolas du 4 juin 1993 ;John Markoff, Abolition of Feudalism : Peasants, Lords and Legislators in the French Revolution, University Park, 1996. Mes propres recherches dans les archives couvrent toute la période de la fin du xvie siècle à la seconde moitié du xixe siècle. J'ai effectué des recensements d'émeutes pour chaque période. Par rapport aux études de J. Nicolas et J. Markoff, j'aboutis aux résultats suivants : de 1661 au 30 avril 1789, 1265 émeutes de subsistance ; de 1788 au 30 juin 1793, 1012 émeutes ; 1794-95, 246 ; pour l'ensemble de la période 1585-1799, j'ai recensé 2351 émeutes dont l'approvisionnement en grains (et leurs produits) fut la préoccupation principale et dominante.

4.Florence Gauthier, Guy-Robert Ikni, éd., La guerre du blé au XVIIIe siècle : la critique populaire contre le libéralisme économique, Paris, 1988. 
5.Voir sur ce point, Cynthia Bouton, The Flour War : Gender, Class, and Community in Late Ancien Régime French Society, University Park, PA, 1993.

6.Il y a toujours des difficultés lorsque l'on tente d'esquisser des traits communs à toute la France, surtout avant la Révolution. Des différences profondes distinguent des régions et même de petits pays. Néanmoins, je crois possible, et même important, de tenter des synthèses. Ma méthode ici consiste à repérer des périodes découvrant des traits communs à travers la France, et ensuite de les comparer.

7.Voir sur ce point James Scott, Moral Economy of the Peasant : Rebellion and Subsistence in Southeast Asia, New Haven, 1976. La logique de fond de ce comportement se trouve expliquée par M. Foster, "The Peasants and the Image of Limited Good », American Anthropologist, $\mathrm{n}^{\circ}$ 67, 1965, pp. 293-315. Il ne s'agit pas d'idéaliser cette hypothèse : les inégalités, parfois énormes, dans l'accès à ces ressources nécessaires et le mépris de cette norme pouvaient exister et des sociétés différentes l'expriment de façon particulière. On discute encore de la signification des comportements tels que la " réciprocité » et l'échange de « cadeaux ", voir à ce sujet Bronislaw Malinowski, Crime and Custom in Savage Society, Paterson, N.J., 1926 ; Marcel Mauss, « Essai sur le don : forme et raison de l'échange dans les sociétés archaïques », dans Sociologie et Anthropologie, Paris, 1950, 1995 ; Karl Polanyi, The Great Transformation, Boston, 1957 ; Marshall Sahlins, Stone-Age Economics, New York, 1972, trad. Âge de pierre, âge d'abondance. L'économie des sociétés primitives, Paris, 1972 ; et plus récemment, «Obligation de donner. La découverte sociologique capitale de Marcel Mauss » La Revue du M.A.U.S.S., n ${ }^{\circ}$ 8, 2e semestre 1996 ; Yan Yunxiang, The Flow of Gifts : Reciprocity and Social Networks in a Chinese Village, Stanford, 1996.

8.Pour le Moyen Âge voir Brian Tierney, The Medieval Poor Law, Berkeley, 1959, p. 37. Voir aussi Jean MEUVRET, Le problème des subsistances à l'époque Louis XIV, t. 1, La production des céréales dans la France du XVIIe et du XVIIIe siècles, Paris, 1977, p. 39 ; t. 3, Le commerce des grains et la conjoncture, Paris, 1988, p. 155. Deux messages de la chrétienté médiévale - l'obligation morale de secourir les nécessiteux et la doctrine de l'innocence du « voleur » poussé par la faim - pouvaient renforcer les revendications au droit à la subsistance. G. Couvreur, Les pauvres ont-ils des droits? Recherches sur le vol en cas d'extrême nécessité depuis la Concordia de Gratien (1140) jusqu'à Guillaume d'Auxerre, Rome, 1961 ; Thomas d'Aquin, Summa Theologiae, II, 66, 7 ; Tierney,op. cit., pp. 37-38, 39, 117. À voir aussi, S. G. Swanson, «The Medieval Foundations of John Locke's Theory of Natural Rights : Rights of Subsistence and the Principle of Extreme Necessity ", History of Political Thought 18 :3, Automne 1997, pp. 399-459. Même si l'on peut douter que les paysans aient connu la scholastique, la diffusion de ses propositions est cependant attestée ; le clergé était lui-même responsable de leur légitimation. Voir par exemple le prône de l'évêque de Strasbourg en 1481, exhortant ses paroissiens à aller « dans les maisons des riches qui ont du blé : si elles sont fermées, enfoncez les portes à coups de hache, prenez du blé et faites une marque ; que si vous perdez votre marque, venez chez moi, je vous dirai comment vous devez vous justifier ». Les forces de l'ordre lui firent des remontrances. L. Dacheux, Un Réformateur catholique à la fin du Xve siècle. Jean Geiler de Kaysersberg, Paris, 1876, p. 528.

9.Sur l'histoire des marchés voir William Parker et Eric Jones, European Peasants and their Markets : Essays in Agrarian Economic History Princeton, 1975 ; Raymond de Roover, "The Concept of the Just Price : Theory and Economic Politics ", Journal of Economic History 18, décembre 1958, p. 421 ; Abbot P. Usher, The History of the Grain Trade in France, 
1400-1700, Cambridge, MA, 1913 ; Dominique Margairaz, Foires et marchés dans la France préindustrielle, Paris, 1988 ; J. Meuvret, Le problème des subsistances..., op. cit., t. III, pp. 97-187 ; George Grantham, « Jean Meuvret and the Subsistence Problem in Early Modern France ", Journal of Economic History, n 49, 1989, pp. 190-92.

10.Amartya Sen, Poverty and Famines : An Essay on Entitlement and Deprivation, Oxford, 1981.

11.Edward Palmer Thompson, « The Moral Economy of the English Crowd in the Eighteenth Century ", Past and Present, 50, 1971, pp. 76-136, trad. en français, "L'Économie morale de la foule dans l'Angleterre du XviIIe siècle », in La Guerre du blé..., op. cit., pp. 31-92.

12. Ce que Pierre Bourdieu a appelé « un consensus sur le sens du monde », Esquisse d'une théorie de la pratique, Genève, 1972.

13.Sur ces régulations, qui comprenaient ce qu'on appelait « la police des grains », voir, par exemple, Pierre Binet, La Réglementation du marché du blé en France au XVIIIe siècle et à l'époque contemporaine, Paris, 1939 ; Léon Biollay, Études économiques sur le XVIIIe siècle. Le pacte de famine. L'administration du commerce, Paris, 1885, pp. 1-22 ; Usher, History of the Grain Trade, op. cit.; J. Martineau, Les halles de Paris des origines à 1789 : évolution matérielle, juridique et économique, Paris, 1960.

14.E. P. Thompson, « Folklore, Anthropology, and Social History », Indian Historical Review, January, 1978 ; « Eighteenth-Century English Society : Class Struggle without Class », Social History, mai 1978, pp. 133-66, et particulièrement page 150 où il appelle cette relation le « patronage-deference equilibrium ».

15.Thompson considère que « la foule tirait en fait son sentiment de légitimité du modèle paternaliste ", «L'économie morale », p. 50. Toutefois, je pense que le peuple et les autorités tiraient la revendication de légimité de la notion originelle du droit à la subsistance qui leur était commune. Certes, le modèle paternaliste était devenu le cadre historique dans lequel s'exprimait l'économie morale de l'Ancien Régime, mais cela n'avait rien à voir avec le point de départ originel de cette notion, voir aussi, John Bohstedt, Riots and Community Politics in England and Wales, 1790-1810, Cambridge, MA, 1983, pp. 35-37, 66, n. 160. Les anthropologues James Scott et William Roseberry ont apporté des raffinements à la théorie de l'économie morale. Scott suggère qu'il faut la regarder comme une « reconstitution imaginative du passé au service des intérêts actuels » et Roseberry propose qu'on la considère comme un « mythe mobilisable » qui n'avait jamais existé dans le détail, mais qui fonctionnait comme une force capable d'influencer le présent et de légitimer les revendications actuelles en faisant allusion au passé ; Scott, Weapons of the Weak : Everyday Forms of Peasant Resistance, New Haven, 1985, p. 305 ; Roseberry, Anthropologies and Histories : Essays in Culture, History and Political Economy, New Brunswick, N.J., 1989., pp. 55-58 ; Bouton, Flour War..., op. cit., pp. 35, 165-67.

16.Voir les émeutes de 1316 et 1322 décrites par Michel Mollat et Philippe Wolff, Ongles bleus, Jacques et Ciompi. Les Révolutions populaires en Europe aux XIVe et Xve siècles, Paris, 1970 , et les difficultés des XIVe et XVe siècles décrites par L. Stouff, Ravitaillement et alimentation en Provence aux XIVe et XVe siècles. Civilisations et Sociétés, 20, Paris, 1970, pp. 72-81.

17.Voir parmi les synthèses récentes, D. Potter, A History of France, 1460-1560: Emergence of a Nation State, New York, 1995, pp. 11-16 ; Arlette Jouanna, La France du XVIe siècle, 1483-1598, Paris, PUF, 1996, pp. 26-36, 102 ; voir aussi P. Chaunu, R. Gascon, E. Le Roy 
Ladurie, M. Morineau, Histoire économique et sociale de la France, Paris, 1977, t. 1, L'État et la ville. Paysannerie et croissance. Les années 1520, marquées par la famine dans le nord du Royaume en 1521 et la Grande Rebeyne à Lyon en 1529, furent difficiles, mais ne sont pas comparables aux crises plus profondes de la seconde moitié du siècle. 18. Charles Tilly, "Food Supply and Public Order in Modern Europe », The Formation of National States in Western Europe, ed. C. Tilly, Princeton, 1975, pp. 380-455.

19.Voir Jouanna, op. cit., pp. 542-554, 619-632 ; P. Benedict, « Civil War and Natural Disaster in Northern France », et M. Greengrass, " The later Wars of Religion in the French Midi », dans P. CLARK ed., The European Crisis of the 1590s, London, 1985, pp. 84-105, 106-134.

20.Philip T. Hoffman, Growth in a Traditional Society : The French Countryside, 1450-1815, Princeton, 1996, p. 100.

21.Voir les prix de 300 à $450 \%$ au-dessus du prix courant après 1630 par rapport à une hausse antérieure de 40 à 80 \%, Grantham, « Jean Meuvret », art. cit., p. 187.

22.Id., p. 100.

23.BNF, Mélanges Colbert, 107-109.

24.Ibid.

25. "Economic Crises and Social Atmosphere in Urban Society under Louis XIV », dans State and Society in Seventeenth-Century France, ed. R. Kierstead, New York, 1975, p. 239, trad. de «Crises économiques et atmosphère sociale en milieu urbain sous Louis XIV », Revue d'Histoire Moderne et Contemporaine, $\mathrm{n}^{\circ}$ 14, 1967.

26.En 1662, les échevins de Blois se plaignirent des comportements des marchands. Ils déclarèrent : " c'est que ayant voulu traiter doucement et favorablement on leur (les marchands) a laissé la liberté de mettre eux-mêmes le prix à leurs marchandises qui a été si excessif se prevalent de notre extrême necessité que les deux tiers du peuple n'a pas eu le moyen de s'en pouvoir munir ", Lettre des échevins de Blois à Colbert, 26 mai 1662, BNF, Mélanges Colbert, 109, n. 778. Sur la crise des années 1690, voir Patrice Berger, « The Famine of 1692-1694 in France : A Study in Administrative Response ", Ph.D. dissertation, University of Chicago, 1972, p. 209.

27.La monarchie déclara qu'elle invitait les marchands à ne pas trop profiter des disettes pour faire monter excessivement les prix (Berger, op. cit., p. 202).

28.On ne prend pas suffisamment en compte ce rôle de l'État dès lors que l'on privilégie son rôle paternaliste en se fiant seulement à sa rhétorique en faveur des consommateurs.

29.W. Beik, Urban Protest in Seventeenth-Century France : The Culture of Retribution, Cambridge, 1997, p. 71.

30.Pendant les années 1660, Louis XIV fit des efforts pour approvisionner Paris, en faisant venir des grains de Danzig, construire des fours aux Tuileries et distribuer du pain à un prix bas qu'il fixa (Nicolas Delamarre, Traité de police, II, pp. 1022-1037 ; Gustave Bord, Histoire du blé en France. Le pacte de famine, histoire, légende, Paris, 1887, pp. 21-22). Pour les XIVe et XVe siècles, voir Stouff, Ravitaillement et alimentation en Provence..., op. cit., pp. 72-74. Récemment, K. G. Persson a souligné que l'intervention gouvernementale dans le commerce des grains avait été une réponse efficace et cohérente dans le cadre d'une économie préindustrielle comme celle que connaissait la France : « The seven lean years, elasticy traps, and intervention in grain markets in pre-industrial Europe », Economic History Review, 49, 1996, pp. 692-714. 
31.Robert A. Schneider, Public Life in Toulouse, 1463-1789: From Municipal Republic to Cosmopolitan City, Ithaca, 1989, p. 321.

32.En 1587, par exemple, les autorités d'Abbeville arrêtèrent une cargaison de grains destinée à Amiens et en vendirent une partie aux habitants de la ville, voir MarieLouise Pelus-Kaplan, « Une crise de subsistances à Amiens (1586-1587) », Annales Historiques Compiègnoises, $\mathrm{n}^{\circ}$ 15, 1981, p. 10.

33.Mais pratiquement nulle part ailleurs, voir Hoffman, Growth in a traditional society..., op. cit., pp. 132-133.

34.L. Dermigny dressa « une carte monétaire » de la France à la fin du XVIIIe siècle qui montre « que la France n'est pas une, mais en partie double » : une France de l'or, signe de croissance d'une économie du commerce maritime, colonial et national et une France d'argent, signe d'une économie plutôt locale, ou peut-être régionale, en tous cas plus restreinte (dans « Une Carte monétaire de la France à la fin de l'Ancien Régime », Annales ESC, 10, 4, 1955, pp. 480-93). Voir aussi Ernest Labrousse, Esquisse du mouvement des prix et des revenus en France au XVIIIe siècle, Paris, 1933. Une lettre de 1773 d'un curé du village de Grateloup près Tonneins offre un exemple des conséquences d'une disette causée par le commerce des grains. Il expliqua « qu'il y a cependant partout... autant de blé qu'il en faut pour nourrir tous les habitants des pays soulevés ; c'est l'argent seul qui y manque pour acheter » (lettre du 23 mai 1773, AD Gironde, C 660). Voir aussi la situation qui favorisa le marché de Toulouse, Georges Freche, « Études statistiques sur le commerce céréalier de la France méridionale au XVIIIe siècle ", Revue d'Histoire Economique et Sociale, 49, 1971, pp. 1-26. Sur la persistance des marchés de blé segmentés au XVIIIe siècle et même au XIXe siècle, voir J.M. Chevet et P. Saint-Amour,

"L'Intégration des marchés de blé en France aux XVIIIe et XIXe siècles », Cahiers d'Économie et de Sociologie Rurales, 22, 1992, pp. 152-175 et J.M. Chevet, « National and Regional Corn Markets in France from the Sixteenth to the Nineteenth Century ", Journal of European History, 25, 3, 1996, pp. 681-703

35.James Collins, The State in Early Modern France, Cambridge, 1995, p. 179. Sur la question du développement des marchés, voir Louise Tilly, «The Food Riot as a Form of Policial Conflict in France », Journal of Interdisciplinary History 2, 1971, pp. 23-57, trad. en français, Annales ESC, 3, 1972, pp. 731-757 ; et David WEIR, « Markets and mortality in France, 1600-1789 » in Famine, Disease and the Social Order in Early Modern Society, eds. J. Walter et R. Schofield, Cambridge, 1989.

36. Olwen Hufton résume ainsi : « la famine périodique fut remplacée pour à peu près 30 à $40 \%$ de la population globale, peut-être 60 à $70 \%$ dans quelques régions, par une malnutrition à long terme ", " Social Conflict and the Grain Supply in EighteenthCentury France », Journal of Interdisciplinary History, 14, 2, 1983, p. 305. Voir aussi Robert Schwartz, Policing the Poor in Eighteenth-Century France, Chapel Hill, 1988, pp. 132-53.

37.Labrousse montre que la deuxième moitié du XvıIIe siècle connaissait des mouvements inégaux des prix, des salaires, et des rentes (Esquisse..., op. cit., pp. 103-13, 176, 183). Voir la critique récente de Michel Morineau, "Réponse à Guy Lemarchand ", $A H R F, 305,1996$, pp. 528-30. Hoffman a observé récemment que pendant le XVIIIe siècle, les crises de subsistances diminuaient peu à peu, mais le niveau de vie stagnait. Les prix des subsistances montaient et les salaires réels, même s'ils diminuaient moins que ne l'ont pensé les historiens, ne pouvaient se maintenir au niveau, Growth in a Traditional Society..., op. cit., p. 135. 
38.Vers les années 1760 et 1770 les structures de l'assistance des pauvres connurent d'importantes crises (Camille Bloch, L'Assistance et l'État en France à la veille de la Révolution, 1764-1790), Paris, 1908 ; Jean-Pierre Gutton, La Société et les pauvres : l'exemple de la généralité de Lyon, 1534-1789, Paris, 1970 ; Michel Vovelle, Piété baroque et déchristianisation en Provence au dix-huitième siècle, Paris, 1973 ; Olwen H. Hufton, The Poor in Eighteenth-Century France, 1750-1799, Oxford, 1974 ; Cissie Fairchilds, Poverty and Charity in Aix-en-Provence, 1640-1789, Baltimore, 1976 ; Colin Jones, Charity and «Bienfaisance » : The Treatment of the Poor in the Montpellier Region, 1740-1815, Cambridge, 1982 ; Thomas Adams, Bureaucrats and Beggars : French Social Policy in the Age of Enlightenment, New York, 1990 ; Catherine Duprat, Le temps des philanthropes : La philanthropie parisienne des Lumières à la monarchie de Juillet, t. 1, Paris, 1993.

39. Simone Meyssonnier, La Balance et l'horloge : La genèse de la pensée libérale en France au XVIIIe siècle, Paris, 1989.

40.Voir à ce sujet Judith Miller, Mastering the Market : The State and the Frain Trade in Northern France, 1700-1860, Cambridge, 1999, pp. 50-92.

41.The Political Culture of the French Revolution, ed. Colin Lucas, London, 1988 ; Jeffrey Merrick, The Desacralization of the French Monarchy in the Eighteenth Century, Baton Rouge, 1990 ; S. Maza, Private Lives and Public Affairs. The Causes Célèbres of Pre-Revolutionary France, Berkeley, 1993 ; Steven Kaplan, The Famine Plot Persuasion in Eighteenth Century France, Philadelphia, 1982, trad. Le Complot de famine. Histoire d'une rumeur au XVIIIe siècle, Paris, 1982.

42.Steven Kaplan, Bread, Politics and Political Economy in the Reign of Louis XV, 2 vol., The Hague, 1976, II, p. 488, trad., Le Pain, le Peuple et le Roi, Paris, 1986.

43.Mais le gouvernement lui-même n'hésitait pas à taxer le prix du pain quand il le jugeait nécessaire, voir Judith Miller, « Politics and Urban Provisioning Crises : Bakers, Police and Parlements in France, 1750-1793 », Journal of Modern History, 64, 1992, pp. 227-262. Le gouvernement décida que les autorités pourraient recourir à la persuasion, mais ni à la force ni aux menaces, J. Miller, Mastering the Market: The State and the Grain Trade in Northern France, 1700-1860, op. cit., p. 78.

44.Cynthia Bouton, « Les Victimes de la violence populaire pendant la Guerre des farines, 1775 », dans Mouvement populaire et conscience sociale, XVIe-XIXe siècle, éd. J. Nicolas, Paris, 1987, pp. 391-99 ; “"L'économie morale” et la Guerre des farines », dans La Guerre du blé..., op. cit., pp. 105-6 ; Flour War..., op. cit., pp. 235-249.

45. Colin Lucas, "The Crowd and Politics in France ", Journal of Modern History 60, septembre 1988 , p. 430.

46.S. Reinhardt, Justice in the Sarladais, 1770-1789, Baton Rouge, 1991, p. 26. Voir aussi la correspondance sur le déploiement des troupes dans la région de Bordeaux en juin 1773 (lettres des 6 et 7 juin, AD Gironde, C 1435).

47.Procès-verbal de l'assemblée de police de Reims, 11 juillet 1770 et Lettre du procureur général du parlement de Paris, 12 juillet 1770 (BNF, Collection Joly de Fleury, 1151, nos. 161-165, 174-175) ; R. B. Rose, « Eighteenth-Century Price Riots, the French Revolution, and the Jacobin Maximum », International Review of Social History 4, 1959, pp. 433-434 ; Kaplan, Le Pain, le Peuple et le Roi, op. cit., p.312 ; B. Vonglis, Le Commerce des céréales à Reims au XVIIIe siècle, Reims, 1980, pp. 227-231; Georges Boussinesq et Gustave Laurent, Histoire de Reims, Reims, 1980, pp. 247-249.

48.Archives historiques de la guerre (AHG), A1 3694 ; lettre de M. de Pommery à l'intendant Crosne, 10 mai 1775 (AD Seine-Maritime, C 108, n. 108) ; G. Schelle, Cuvres de 
Turgot et documents le concernant, 5 vol., Glashutten im Taunus, 1972, IV, p. 49, n. 2 ; Édgar Faure, La Disgrâce de Turgot, Paris, 1961, pp. 268-79 ; Rapport sur l'émeute arrivée à Paris par rapport aux prix du bled et du pain le 3 mai 1775 et différentes pièces relatives à cet événement (AN, H2* 1879), voir aussi AN, K 1022.

49.Le 4 mai, le roi réquisitionna les troupes. Il dépêcha deux armées pour patrouiller à Paris, dans les banlieues, et le long de la Seine et donna le commandement au maréchal Biron. L'exemple de Montlhéry, gros marché de grains, souligne le rôle important des troupes. Les forces de l'ordre qui arrivèrent le 8 mai comprenaient 50 grenadiers du régiment des gardes et environ 40 grenadiers royaux du régiment de Paris. Le maréchal de Poyanne expliqua les mesures qu'il prenait : «J'ai fait fermer toutes les avenues qui n'étaient pas absolument nécessaires, et occuper celles qui resteraient libres. J'ai placé des postes dans tous les autres endroits que j'ai jugé plus convenables à contenir ces gens-là, et j'ai fait publier qu'on ferait tirer sur tous ceux qui oseraient troubler l'ordre et la tranquilité du marché. Il est venu un monde immense... il y a eu quelques murmures de la part de ce peuple en demandant le bled à 12 livres ainsi qu'ils sont dans l'usage de le faire, mais ils n'ont rien osé entreprendre » (lettre de Poyanne, 8 mai 1775, AHG, A1 3694, nos 86-87).

50.Bouton, Flour War..., op. cit., pp. 95-96.

51.Un arrêt du conseil d'état du roi « portant attribution aux prévôts des maréchaussées et leur lieutenants, chacun dans son département pour connaître des séditions, émotions et attroupements qui pourraient survenir à l'occasion des bleds et autres grains » du 19 mai 1739 (AD Indre-et-Loire, C 94), essaya de confier tous les procès aux prévôtés, mais ce ne fut que pendant la Guerre des farines que la monarchie y parvint. Sur les cours prévôtales voir Reinhardt, Justice in the Sarladais..., op. cit., p. 69 ; Nicole Castan, Les Criminels de Languedoc : les exigences d'ordre et les voies du ressentiment dans une société prérévolutionnaire. 1750-1790, Toulouse, 1980 ; Julius Ruff, Crime, Justice and Public Order in Old Regime France: The Sénéchaussées of Libourne and Bazas, 1696-1789, London, 1984 ; M. Rateau, « Les peines capitales et corporelles en France sous l'Ancien Régime, 1670-1789 ", Annales internationales de criminologie (1963), pp. 281-282 ; J. Carey, Judicial Reform in France before the Revolution of 1789, Cambridge, MA, 1981, p. 21.

52.Florence Gauthier, « Robespierre, critique de l'économie politique tyrannique et théoricien de l'économie politique populaire ", dans Robespierre : de la nation artésienne à la République et aux nations: Actes du colloque d'Arras, 1-3 avril 1993, Lille, 1994, p. 237. Robespierre fait le lien entre la liberté du commerce des grains et la répression : « la liberté indéfinie du commerce et des baïonnettes pour calmer les alarmes ou pour apaiser la faim » («Sur les subsistances », discours à la Convention, 2 décembre 1792, Robespierre, Euvres, t. 9, Paris, 1958, p. 111).

53.Kaplan, Le Pain ;..., op. cit. J. Miller, Mastering the Market..., op. cit., p. 80, précise que même les autorités comprenaient mal ce que signifiait la liberté du commerce.

54.Selon le subdélégué de Gournay-en-Bray qui écrit après une émeute de mai 1775 : «Quand nous disons au peuple que la révolte du 2 mai 1775 a empêché les laboureurs d'apporter depuis ce temps du bled au marché et que c'est là la cause du renchérissement de la denrée, il nous répond que si cette émotion n'était pas arrivée le bled se vendrait à présent 8 livres le boisseau. Au surplus, le calme est rétabli, la liberté est rendue au commerce, nos deux derniers marchés ont été garnis suffisamment ». Le prix au marché précédant l'émeute avait monté jusqu'à 6 livres 2 sols et les émeutiers le fixèrent à 3 livres (lettre du 4 juin 1775, AD Seine-Maritime, C 107). 
55.Kaplan, Bread, II, pp. 700-702 ; Id., Le Meilleur pain du monde : les boulangers de Paris au XVIIIe siècle, Paris, 1996, p. 37.

56. Markoff, Abolition of Feudalism..., op. cit. Pour une étude plus ancienne, mais très utile, voir Anatoli Ado, Paysans en Révolution : Terre, pouvoir, et jacquerie, 1789-1794, trad. du russe, Paris, 1996 (1971-1987).

57.Fernand Gerbaux et Charles Schmidt, ed. Procès-verbaux des comités d'agriculture et du commerce de la Constituante ; de la Législative, et de la Convention, 4 vol., Paris, 1906, I, p. 374 et Miller, Mastering the Economy..., op. cit., p. 183.

58.Tableau de ce qui s'est passé dans cette province à l'occasion des troubles actuels, Orléans (s. d.) (AN, H1 1453). Le jour même du dépôt des cahiers par les trois ordres à Tours, « le peuple se porte en masse au marché des grains et taxe arbitrairement les grains » (H. Faye, La Révolution au jour le jour en Touraine (1789-1800), Angers, 1903, p. 10). Les 3 et 4 mai 1789, " le peuple » de Limoux en Languedoc, "sous prétexte de faire la recherche des grains qui peuvent exister dans la communauté chez les fermiers de la subvention et de l'équivalent, ainsi que chez le contrôleur des actes... a emporté de ces différentes maisons les registres » (Tableau des troubles arrivés dans la province de Languedoc, s.d., AN, H1 1453).

59.M. Bruneau, Les Débuts de la Révolution dans les départements du Cher et de l'Indre, 1789-1791, Paris, 1902, réed. Genève, 1977, pp. 49-50. Voir aussi une attaque semblable à Moulins le 23 août 1789, lorsqu' " un commerçant riche entra en ville... le peuple crut voir en lui un de ces accapareurs de bled qui par des achats odieux lui dévorent sa subsistance » (Mémoire, septembre 1789, AN, BB 30 79).

60.Louis de Cardenal, « Les Subsistances dans le département de la Dordogne, 1789-an IV », La Révolution française, $\mathrm{n}^{\circ} 82,1929$, p. 218.

61.F. Mourlot, La Fin de l'Ancien Régime dans la généralité de Caen. 1787-1790, Paris, 1913, p. 313 ; Rose, op. cit., p. 437. L'émeute de Bourges se termina par 67 arrestations, trois hommes furent condamnés à 9 ans de galères, un à 3 ans, et une femme à 9 ans de prison (Bruneau, Les Débuts de la Révolution..., op. cit., p. 50). Néanmoins, le procureur du roi avait demandé la peine de mort contre trois de ces personnes, dont une femme. 62.Lettre du 15 avril 1789, AD Indre-et-Loire, C 99. Voir aussi Guy Lemarchand, La Fin du Féodalisme dans le pays de Caux, Paris, 1989, p. 417.

63.Lettre des officiers municipaux d'Orléans au contrôleur général, 28 avril 1789 (AN, H1 1453) et Georges Lefebvre, Études Orléanaises : Contribution à l'étude des structures sociales à la fin du XVIIIe siècle, 2 vol., Paris, 1962, II, pp. 19-20, 33. Des soldats tuèrent un homme et blessèrent plusieurs autres personnes pendant une émeute à Yvetot en mars (Lemarchand, Fin du Féodalisme..., op. cit., p. 417).

64.Décret du 10 août 1789 (Archives Parlementaires, t. 8, pp. 376-379) et la loi martiale du 21 octobre 1789 (AP, t. 9, pp. 475-476). L'Assemblée vota la loi martiale à la suite d'émeutes frumentaires à Paris pendant lesquelles les émeutiers pendirent un boulanger (George Rudé, The Crowd in the French Revolution, London, 1959, trad. La Foule dans la Révolution française, Paris, 1982, p. 97). Voir aussi Florence Gauthier, Triomphe et mort du droit naturel en Révolution, 1789, 1795, 1802, Paris, 1992, pp. 56-66.

65.Voir la description du comportement des autorités dans les municipalités de Normandie pendant la crise de 1789, Judith Miller, Mastering the Market..., op. cit., pp. 181-190, qui suggère que les comportements des autorités s'expliquent plutôt par l'inexpérience que par l'idéologie, mais leur langage évoquait fréquemment le droit à la subsistance et la fraternité, pp. 189-190. 
66. Howard Brown, War, Revolution and the Bureaucratic State : Politics and the Army Administration in France, 1791-1799, Oxford, 1995, pp. 38-71.

67.Voir les cartes et tableaux de John Markoff, Abolition of Feudalism, op. cit., chap. 7, pp. 337-368, qui complètent ceux de Anatoli Ado, Paysans en Révolution..., op. cit. Pendant cette période, les troubles de subsistance accompagnaient les jacqueries, les mouvements anti-fiscaux, religieux ou contre-révolutionnaires.

68.Francis Évrard, «Les subsistances en céréales dans le département de l'Eure de 1788 à l'anV », Bulletin trimestriel de la Commission de recherche et de publication des documents relatifs à la vie économique de la Révolution, Paris, 1909, pp. 42-43; Michel Vovelle, « Les taxations populaires de février-mars et novembre-décembre 1792 dans la Beauce et sur ces confins ", dans Ville et campagne au XVIIIe siècle : Chartres et la Beauce, Paris, 1980, pp. 259-63. Voir aussi Rudé, Crowd in History. A Study of Popular Disturbances in France and England, 1730-1848, 1964, London, 1964, réed. 1985, pp. 110-111 ; Guy-Robert Ikni, « L'arrêt des bateaux de grains sur l'Oise et l'Aisne en février 1792 », Annales historiques compiègnoises modernes et contemporaines, 5, janvier 1979 ; Albert Mathiez, La vie chère et le mouvement social sous la Terreur, Paris, 1927, réed. 1973, pp. 62-63.

69.La politique économique populaire ne se développait pas partout de la même manière et exprimait des nuances diverses qui dépendaient de plusieurs éléments : les structures et relations sociales, les traditions culturelles et les rapports politiques, par exemple. Mais on discerne une idée générale de cette politique de subsistance dès 1792 . Sur le pouvoir rhétorique de la langue du droit aux subsistances, voir William Sewell, "The Sans-culottes Rhetoric of Subsistence », dans Keith Baker ed., The Terror. The French Revolution and the Creation of Modern Political Culture, Oxford, Pergamon, vol. 4, 1994, pp. 249-269. Voir aussi Michael Sonenscher, Work and Wages : Natural law, Politics and the Eighteenth-century French Trades, Cambridge, C. University Press, 1989, pp. 353, 358. Toutefois, je ne partage pas l'opinion de ces auteurs qui estiment que la crise de subsistance, à cette époque, ne menaçait pas l'existence de la plupart de ceux qui se nommaient « Sans-culottes ».

70.Sur l'émergence du droit à l'existence dans la Révolution et l'élaboration du programme du Maximum, voir Albert Soboul, Les Sans-culottes parisiens en l'an II, Paris, 1958, IIe partie, pp. 404-677 ; George Rudé, The Crowd..., op.cit. ; Richard Cobb, Les Armées révolutionnaires, instrument de la Terreur dans les Départements, Paris, 2 vol., 1961, 1963 ; Kare Tönnesson, La Défaite des Sans-culottes, Paris-Oslo, 1959, réed. 1978 ; A. Soboul ed., Girondins et Montagnards. Actes du Colloque, Sorbonne, 1975, Paris, Société des études robespierristes, 1980. Vu l'ampleur du sujet qui dépasse le cadre de cette étude, je m'en tiendrais ici à une rapide esquisse en m'appuyant sur les travaux les plus récents. 71.Bryant Ragan, Rural Political Culture in the Department of the Somme during the French Revolution, Ph. D. dissertation, University of California at Berkeley, 1988, pp. 128-129. 72.Interrogatoire d'Alexandre Cocardat, 24 mai 1792 (AD Yvelines, 42 L 27) ; Sukla Sanyal, Riots and Revolution : Food riots in the department of the Seine-et-Oise, 1789-1795, Ph.D. dissertation, University of Maryland, 1994, p. 336.

73.Extrait du procès-verbal du 24 mars 1792 (AD Yvelines, 1 LM 460).

74.En février 1793, la Société populaire de Chalon-sur-Saône diffusa une adresse proposant de faire des grains des " propriétés nationales », voir R. Carraz, "Girondins et Montagnards, le cas chalonnais", dans Girondins et Montagnards, op. cit., pp. 167-192. 75.Par exemple, à Chevreuse, Seine-et-Oise : A. Defresne et F. Évrard, Les subsistances dans le district de Versailles de 1788 à l'an V, 2 vol., Rennes, 1921, I, p. 237 ; Sanyal, op. cit., p. 86. À Falaise dans l'Oise, Ikni, Crise agraire et Révolution paysanne : le mouvement populaire 
dans les campagnes de l'Oise de la décennie physiocratique à l'an II, thèse de doctorat d'État, Université de Paris I, 1993, 3e Partie, p. 300, n 73. À Jagny, Seine-et-Oise, Daisy Guglielmetti, « Les troubles de subsistance de 1790-1792 dans le district de Gonesse », dans Les Paysans et la Révolution en pays de France. Actes du Colloque de Tremblay-lès-Gonesse, octobre 1988, Paris, 1989, p. 144. À Longjumeau, Seine-et-Oise, Defresne et Évrard, op. cit., I, p. 236. À Montlhéry, Seine-et-Oise, Sanyal, op. cit., p. 72.

76.Sur l'importance des districts pour l'approvisionnement, voir R. Cobb, Terreur et subsistances. 1793-1795, Paris, 1964, pp. 213-214 ; Woloch, The New Regime. Transformations of the French Civic Order, 1789-1820s, New York, 1994, pp. 38-39.

77.Lettre du 7 août 1793 (AD Seine-Maritime, L 1387).

78.Extrait des registres du conseil du département de la Loire-Inférieure des 8 et 17 octobre 1792, AN, F11 214. Le commissaire était arrivé avec une force armée impressionnante : 280 hommes, dont 200 fantassins, 40 cavaliers, et 50 artilleurs (sic). À leur arrivée, le commissaire et son armée rétablirent l'ordre à coups d'arrestations et imputèrent à la commune les frais de déplacement des troupes, soit une somme de 1822 livres.

79.Georges Lefebvre, Documents relatifs à l'histoire des subsistances dans le district de Bergues pendant la Révolution, 1788-an V, Lille, 2 vol. 1914-1921, 1, pp. 226-273. G. Carrot, Révolution et maintien de l'ordre, 1789-1799, Paris, 1995, p. 211 ; Mathiez, La vie chère..., op. cit., p. 60.

80.Décret qui proclame une amnistie et déclare « éteints et abolis » tous les procès criminels et jugements qui avaient été intentés ou prononcés depuis le 14 juillet 1789 pour violation des lois relatives aux grains et aux biens communaux, 3 septembre 1792. R. Werner, L'Approvisionnement en pain de la population du Bas-Rhin et de l'armée du Rhin pendant la Révolution. 1789-1797, Strasbourg, 1951, p. 125. Voir aussi Archives parlementaires, t. 49, pp. 240-241. Seuls ceux qui avaient donné ou reçu de l'argent furent exclus de cette amnistie.

81.Décret relatif aux auteurs, fauteurs, et complices des attroupements pour s'opposer à la libre circulation des grains et aux dénonciateurs, 6 décembre 1792 (AN, AD XI 69) ; Décret qui établit la peine de mort contre les personnes qui s'opposeraient au libre accès des subsistances à Paris, 6 décembre 1792 (AD Tarn, L 344) ; Décret sur les subsistances, 8 décembre 1792 (« Recueil des principaux textes législatifs et administratifs sur le commerce des céréales de 1788 à l'an V », Bulletin de la Commission de recherche et de publication des documents relatifs à la vie économique de la Révolution, n. 1, Paris, 1906, pp. 147-148). Voir aussi F. Gauthier, G. R. Ikni ed., La Guerre du blé..., op. cit., pp. 111-167.

82.Décret qui abolit toutes procédures criminelles pour délits commis dans les insurrections qui ont eu lieu relativement aux subsistances jusqu'au 21 janvier dernier, 11 février 1793 (AN, F11 266).

83.Jean-Pierre Gross, Fair Shares For All : Jacobin egalitarianism in practice, London, 1997, pp. 3, 10, 201.

84.Voltaire, « Égalité » Dictionnaire philosophique portatif, Genève, 1764, (1769), réed., Paris, 1964, p. 171.

85.Gross, Fair Shares..., op. cit., p. 201.

86.Id., « L'Économie fraternelle » : les greniers d'abondance de l'an II », Colloque Mably: la politique comme science morale. Actes du colloque, 6-8 juin 1991, 2 vol., Bari, 1997, t. 2, p. 244.

87.Richard Cobb, Les Armées révolutionnaires..., op. cit., Paris, 2 vol., 1961, 1963. 
88. Mathiez, La vie chère ..., op. cit., pp. 289-90 ; Werner, L'approvisionnement en pain..., op. cit., pp. 226, 297, 300, 311 ; Gross, Fair Shares..., op. cit., pp. 64-92 et « L'Économie fraternelle... « , op. cit. ; Lemarchand, La Fin du féodalisme..., op. cit., pp. 479-488; Ikni, «Crise agraire et Révolution... », op. cit. ; Miller, Mastering the Market..., op. cit., ch. 6. 89.Voir par exemple l'émeute à Meures, dans la Marne, en mars 1794 (C. Lorain, Les Subsistances en céréales dans le district de Chaumont de 1788 à l'an V. Documents publiés, Chaumont, 2 vol., 1911, 1, nos 846, 849, 867). L'administration du Maximum rencontra d'immenses difficultés, voir Judith Miller, Mastering the Market..., op. cit., pp. 155-162. Mais en fait, il y eut très peu de troubles durant la période d'application du Maximum et cette politique réussit, au moins, à : « préserver dans l'urgence de l'an II, les fractions les plus démunies et les plus vulnérables de la population » (Dominique Margairaz, « Le Maximum, politique économique ou politique sociale?", dans Robespierre, de la Nation artésienne à la République et aux Nations, Lille, 1994, p. 278), et, au plus, à représenter que le droit à l'existence « doit conditionner l'organisation économique de la société », comme les « droits politiques et civils » (Claude MAZAURIC, « Les choix économiques et sociaux : Préliminaires », id., pp. 231-232).

90.Décret relatif à la suppression de toutes les lois portant fixation d'un maximum sur le prix des denrées et des marchandises, 24 décembre 1794 (Bulletin des lois, an III, nº 538, pp. 1-4). Décret contenant proclamation sur l'abolition des lois relatives au maximum, 29 décembre 1794 («Recueil des principaux textes législatifs et administratifs sur le commerce des céréales de 1788 à l'an V », Bulletin de la Commission de recherche et de publication des documents relatifs à la vie économique de la Révolution, $\mathrm{n}^{\circ} 1$, Paris, 1906, p. 236). Mais, bien avant décembre 1794, la Convention avait déjà laissé s'affaiblir ces formes d'approvisionnement, voir J. Miller, Mastering the Market..., op. cit., p. 169.

91.Pourtant, ce n'était pas compris par toutes les autorités locales. Une lettre du district d'Albi à toutes ces municipalités les avertit que « l'exercice du droit de réquisition ne peut plus être pratiqué à l'avenir, ce qui doit vous prouver que toutes demandes de secours en grains que vous pouviez nous faire resteraient sans effet puisque chaque citoyen doit s'approvisionner par la voie du commerce " (Registre des lettres écrites par le district d'Albi : Lettre aux municipalités du district du 1er pluviôse an III-20 janvier 1795, AD Tarn, L 727*).

92.La Convention maintint les réquisitions. La loi prorogea jusqu'au 1er messidor le délai fixé par la loi du 4 nivôse pour l'approvisionnement des marchés par la voie de la réquisition (9 mars 1795, Bulletin des lois, an IV, n. ${ }^{\circ}$ 691). Le même mois, le Comité de salut public affirma que « les grains dont peuvent disposer les cultivateurs n'appartiennent pas plus aux habitants de leur commune ou de leur district qu'à tous les autres citoyens de la République » (Arrêté du Comité de salut public sur les demandes de dégrèvement ou d'annulation concernant des réquisitions en grains, 23 ventôse an III-13 mars 1795, « Recueil des principaux textes législatifs et administratifs sur le commerce des céréales de 1788 à l'an V », Bulletin de la Commission de recherche et de publication des documents relatifs à la vie économique de la Révolution, $\mathrm{n}^{\circ} 1$, op. cit., p. 245). Le 24 mars 1795, on arrêta une dernière réquisition (Arrêté du Comité du salut public portant réquisition du cinquième des grains, farines et légumes secs existant dans les départements, districts, et communes, affectés à l'approvisionnement des armées et de la commune de Paris, 3 germinal an III-23 mars 1795, op. cit.., pp. 246-247). Les lois qui suivirent en juillet et septembre 1795 réaffirmèrent que les grains et farines devaient être vendus sur les marchés publics, que tout vendeur serait muni d'une patente et que 
les autorités pouvaient faire des réquisitions. Loi portant établissement de patentes pour l'exercice de toute espèce de commerce, 4 thermidor an III-22 juillet 1795 (Bulletin des lois, an III). Loi sur la police du commerce des grains et l'approvisionnement des marchés et des armées, 7 vendémiaire an III-29 septembre 1795 (Bulletin des lois, an IV, $\mathrm{n}^{\circ}$ 1135). Pour une discussion plus approfondie des vicissitudes des régulations, voir Miller, Mastering the Market..., op. cit., ch. 7.

93.À 8\% de sa valeur nominale en décembre 1794, il s'effondra à moins de $1 \%$ en décembre 1795 ; Colin Jones, The Longman Companion to the French Revolution, London, 1988, p. 237. En février 1796, la Convention supprima l'assignat.

94.À Paris, le prix de grain doubla entre décembre 1794 et janvier 1795. Jean-Pierre Jessenne, Histoire de la France : Révolution et Empire, 1783-1815, Paris, 1993, p. 165, voir aussi, Richard Cobb, « Les disettes de l'an II et de l'an III dans le district de Mantes et la vallée de la basse Seine ", Paris et île-de-France, 1951, n 3, pp. 243-244.

95.Cobb, id., pp. 250-251. L'auteur précise par ailleurs : «En l'an II, on cherche avant tout à assurer aux petites gens un pain bon marché : en l'an III, on s'intéresse aux problèmes de ravitaillement dans la mesure seulement où ils peuvent provoquer des désordres dans la Capitale ; quant au reste, la politique gouvernementale cherchant surtout à rassurer les cultivateurs, il ne peut être question d'appliquer une politique de ravitaillement à base d'égalité sociale ", dans « Les problèmes de subsistances de l'an II et de l'an III : l'exemple d'un petit port normand, Honfleur, 1794-1795 ", Actes du 81e Congrès national des sociétés savantes, Rouen-Caen, 1956, Paris, 1956, p. 316.

96.En juillet 1795, une lettre du directoire du département de la Côte-d'Or affirmait que « le grain est soixante fois plus cher qu'en 1790 » (lettre du directoire du département de la Côte d'Or au Comité de salut public, 6 fructidor an III-23 août 1795, AD Côte-d'Or, L 546).

97.S. Sanyal a remarqué que ce genre de désordre avait prédominé dans les pays de grande culture du département de Seine-et-Oise, Riots and Revolution..., op. cit., pp. 113, 127. Guy Lemarchand a décrit des bandes, venant de plusieurs communes, composées de plus de 500 hommes, femmes et enfants (La Fin du Féodalisme..., op. cit., pp. 516-519). Dans les départements de la Sarthe et de l'Orne, les attroupements ne réunissaient pas autant d'émeutiers que dans ceux de Seine-Inférieure, de l'Eure, ou de Seine-et-Oise, pourtant, les mouvements se ressemblaient beaucoup (voir Christine Peyrard, Les Jacobins de l'Ouest : sociabilité révolutionnaire et formes de politisation dans le Maine et la Basse-Normandie (1789-1799), Paris, 1996, p. 304). Richard Cobb notait que : « poussées par la misère et par la dureté des temps, beaucoup de pauvres gens eurent recours au banditisme ", dans " Politique et subsistances en l'an III : l'exemple du Havre ", Annales de Normandie, mai 1955, p. 155. Sur le rôle à la fois légitime et criminel des bandits voir Eric Hobsbawm, Primitive Rebels : Studies in Archaic Forms of Social Movements in the Nineteenth and Twentieth Centuries, New York, 1959, trad. Les Primitifs de la révolte, Paris, 1968.

98. Richard Cobb, Terreur et Subsistances, 1793-1795, Paris, 1964, pp. 263, 282-285 ; Bryant Ragan, Rural Political Culture..., op. cit., pp. 138-139.

99.Extrait du registre des délibérations et procès-verbaux de la commune de Grenouille, 17 floréal an III-6 mai 1795 (AD Seine-Maritime, L 1597).

100.Sur la violence pendant la Révolution voir les contributions récentes de Philippe Goujard, « La Violence et la Terreur », et de Paolo Viola, « Violence révolutionnaire ou Violence du Peuple en révolution? », dans Michel Vovelle, Antoine de Baecque ed., Recherches sur la Révolution, Paris, 1991, pp. 87- 


\section{RÉSUMÉS}

Dans le cadre d'une vaste enquête sur les troubles de subsistance en France, du xvie siècle à l'Empire, l'auteur propose un tableau synthétique en précisant les conditions qui ont favorisé les émeutes de subsistance, les objectifs et le comportement des émeutiers, la réponse des autorités, de la justice et l'intervention, ou non, des forces armées. Les « rapports de subsistance " sont analysés en fonction des changements intervenus dans les conditions du commerce des grains, des orientations politiques et, plus largement, culturelles.

Durant cette longue période, plus de 2500 émeutes de subsistance ont déjà été recensées. La synthèse ici présentée permet à la fois de prendre la mesure du problème et d'offrir un état exhaustif des recherches menées à ce jour.

Subsistance movements and the Problem of the moral economy under Ancien Régime and the French Revolution.

The author summarizes some of the results of her larger study of subsistence riots in France from the 16th century to the Empire. She discusses the conditions that favored food riots, the objectives and behavior of rioters, the responses of authorities and the courts, and the character of repression, including or not the intervention of troops. The "relations of subsistence" are analyzed in light of such factors as changes in the grain trade as well as in political and cultural orientations. She has examined over 2500 riots in this extended time period. The synthesis presented here permits both a general evaluation of the problem and a presentation of results to this point.

Le sommosse di sussistenza e il problema dell'economia morale sotto l'Ancien Régime e la Rivoluzione francese.

Nell'ambito di una vasta indagine sulle sommosse di sussistenza in Francia dal sedicesimo secolo all'Impero, l'autore propone un quadro sintetico pure precisando le condizioni che hanno determinato le sommosse di sussistenza, gli obiettivi e il comportamento dei ribelli, la risposta delle autorità, della giustizia e l'intervento o no delle forze armate. I "Rapporti di sussistenza" sono analizzati in funzione dei cambiamenti intervenuti nelle condizioni del commercio del grano, degli orientamenti politici e più generalmente culturali.

Durante quel lungo periodo, più di 2500 sommosse di sussistenza sono state censite. La sintesi presentata qui permette nello stesso tempo di prendere la misura del problema e di offrire uno stato delle richerche condotte fino ad oggi.

Dentro de una larga investigación sobre los disturbios de subsistencia en Francia del siglo XVI al Imperio, el autor propone un cuadro sintético, especificando las condiciones que favorecieron los motines, los objectivos y el comportamiento de los sublevados, la contestación de las autoridades, de la justicia, y la intervención de las fuerzas armadas. Los "informes de subsistencia" se analizan en relación con los cambios en las condiciones del comercio de los granos, las orientaciones políticas y culturales. Durante este largo periodo, fueron 2500 los disturbios de subsistencia. Este síntesi s permite un conocimiento del fenómeno y ofrece un estado exhaustivo de las investigaciones.

Die Subsistenzunruben und das Problem der moralischen Wirtschaft zur Zeit des Ancien Régime und der französischen Revolution.

Die Autorin faßt einige der Ergebnisse ihrer umfangreichen Untersuchung über die Subsistenzunruhen in Frankreich vom 16. Jabrhundert bis zum Kaiserreich zusammen. Sie zeigt die Bedingungen, die die Subsistenzaufstände begünstigt haben, die Ziele und das Betragen der 
Aufständischen, die Reaktion der Behörden und der Gerichte, das Eingreifen oder Nicht-Eingreifen des Heers. Die Berichte über die Lage der Lehensmittelversorgung werden ebenso im Lichte der Entwichlung des Kornhandels wie der politischen und kulturellen Orientierungen untersucht. Während dieses langen Zeitraums wurden schon über 2500 Subsistenzunruhen gezählt. Die vorliegende Synthese ermöglicht zugleich eire umfassende Einschätzung des Problems und eine erschöpfende Darstellung der bis heute betriebenen Forschungen über diese Frage.

\section{AUTEUR}

\section{CYNTHIA BOUTON}

Texas A\& M University 